\title{
Modeling and Control of Pharmacodynamics
}

\author{
Carolyn Beck \\ Coordinated Science Lab \& Department of Industrial and Enterprise Systems Engineering \\ University of Illinois at Urbana-Champaign \\ beck3@uiuc.edu
}

\begin{abstract}
Modeling and control of drug dosing regimes are particularly well-suited for applications of control design and analysis techniques. These problems frequently incorporate the use of mathematical models, lending themselves to a large range of model-based control methods. There has been ongoing research aimed at the development of closed-loop drug dosing and delivery regimens in a number of specific medical domains for more than five decades. In this paper, we discuss the development of modeling and control methods aimed at closed-loop delivery of pharmaceutical agents. We focus most of this discussion on the problem of controlling sedation levels during surgical procedures; results from the application of linear parameter varying and robust $\mathcal{L}_{1}$-adaptive modeling and control approaches are presented in some detail.
\end{abstract}

\section{Introduction}

Engineering and technology have played a major role in medical advancements and improvements throughout the past century. Notable recent innovations include robotic surgery, controlled prosthetics, bio-materials and advanced imaging technologies. More specifically, over the past 50 years, contributions to medicine founded in control methods have ranged from the invention of the pacemaker in 1950 and ventricular assist devices in the 1980's, to more recent advances incorporating robotic and image-guided surgery, and the ongoing development of the artificial pancreas. Modeling and control of drug dosing regimes are particularly well-suited for applications of control design and analysis techniques; these problems typically incorporate extensive use of mathematical models lending themselves to a large range of model-based control methods. As it turns out, there has been ongoing research aimed at the development of closed-loop drug dosing and delivery regimens in a number of specific medical domains for decades. For example, delivery of insulin for control of diabetes has been considered for over 50 years [39], with a variety of closed-loop methods considered including on-off control, control-to-range algorithms, proportional-integral-derivative (PID) designs, modelpredictive control (MPC) approaches, and fuzzy-logic methods [78, 22, 9, 81, 8, 10,34]; in particular, see [8] and [34] for surveys of control-relevant issues and design approaches for diabetes treatment. Chemotherapy dosing and timing control methods for treatment of various cancers and HIV have been investigated for close to 40 years, with optimal control methods being most commonly used, and recent efforts including game-theoretic approaches, piecewise-linear and positive systems methods, and MPC approaches [80, 53, 88, $59,37,36]$. And, control of anesthetic dosing during surgery to optimally control sedation level and manage hemodynamic functions has been a research interest for over 50 years, with early applications including onoff and PID control designs, and more recent methods including MPC, robust and adaptive control designs $[12,2,27,28,50,5,62,41]$.

In this paper, we focus on the development of modeling and control methods aimed at closed loop (or semiclosed loop) control of pharmacodynamics. We first overview the classic modeling approaches considered for the purpose of analysis and control in Section 2, and note some challenges associated with these approaches. We then discuss a few recent alternative approaches, from which we proceed with a brief review of results on the more specific problem of modeling and control of anesthetic pharmacodynamics in Section 3. We focus a longer discussion on our results for modeling the response to anesthesia using piecewise-linear and linear

(C) 2015. This manuscript version is made available under the Elsevier user license http://www.elsevier.com/open-access/userlicense/1.0/ 
parameter varying (LPV) models, and compare these to results using the standard modeling approaches on the same data sets. In Section 4, we provide a discussion and some simulation results from applications of recent feedback control approaches for anesthesia delivery. A short summary is given in Section 5. We begin with a discussion of the standard modeling paradigm used in pharmacodynamics, that of compartment models, which captures the basic dose-effect relationships and transport delays expected in administration of pharmaceutical agents, but does not capture drug synergy effects or the response to disturbances.

\section{Pharmacodynamics Models}

The traditional modeling paradigm used to describe the relationships between drug inputs, for example anesthetic agents or insulin, and patient outputs or effects, for example, blood pressure, sedation level or blood glucose concentration, is that of compartment models. These models combine pharmacokinetic and pharmacodynamic properties, offering a continuous profile of a drug's concentration versus time in the patient, which further may be related to the time course of the drug's effect by incorporating an effect compartment. We begin with an overview of compartment models, and then note some alternative model structures used in anesthesia and insulin pharmacodynamic modeling.

\subsection{Compartment Models}

Pharmacokinetic (PK) compartment models are used to model and/or predict the disposition of drug in the body, by modeling the simultaneous diffusion of drug through body tissues and the flow of drug in the blood. The common quote used to describe the purpose of PK models is that they capture "what the body does to the drug". These models are meant to account for mass balances of the drug distribution, relying on an anatomical notion of the relation of tissues to circulating blood for the derivation of models. That is, a series of conceptual compartments, intended to represent the body's tissues and organs grouped roughly in order of decreasing blood flow, is used to describe the interchange of drug within the body. At each compartment, drug flows into the compartment from external inputs, or via transfer from other compartments, or both. Drug flows out of the compartment by transfer to other compartments and/or by elimination via metabolic clearance.

As an example, a three compartment model, shown in Figure 1(a), would be represented mathematically by the state equations

$$
\begin{aligned}
\frac{d x_{1}}{d t} & =I+x_{2} k_{21}+x_{3} k_{31}-x_{1} k_{10}-x_{1} k_{12}-x_{1} k_{13} \\
\frac{d x_{2}}{d t} & =x_{1} k_{12}-x_{2} k_{21} \\
\frac{d x_{3}}{d t} & =x_{1} k_{13}-x_{3} k_{31}
\end{aligned}
$$

where $x_{i}$ is the amount of drug in the $i^{t h}$ compartment, $k_{i j}$ is the distribution transfer rate from the $i^{t h}$ compartment to the $j^{\text {th }}$ compartment, $k_{10}$ is the clearance transfer rate out of the central compartment, and $I$ represents the anesthetic infusion rate into the central compartment. Note that it is naturally assumed that the constraints $x_{i}(t) \geq 0$, for all $t \geq 0$ and all $i$, hold.

The drug is administered to and distributed from the central compartment, which represents the highly perfused tissues, for example the lungs for inhaled anesthetics. The peripheral compartment with volume $V_{2}$ represents a vessel rich grouping of tissues and organs (e.g., brain, liver, etc.), which is assumed to reach steady-state equilibrium quickly. The peripheral compartment with volume $V_{3}$ may then correspond to fatty tissues and other vessel poor tissues and organs which equilibrate slowly (e.g., bones). It is assumed that the infused drug will mix immediately in the volume space of the central compartment (e.g., the heart for intravenous injections and the lungs for inhaled anesthetics). The drug concentration in the central compartment then decreases due to metabolic clearance and distribution to other compartments. The associated transfer rate constants, $k_{i j}$, are determined empirically. For drugs administered by bolus, single compartment models are used most commonly. 

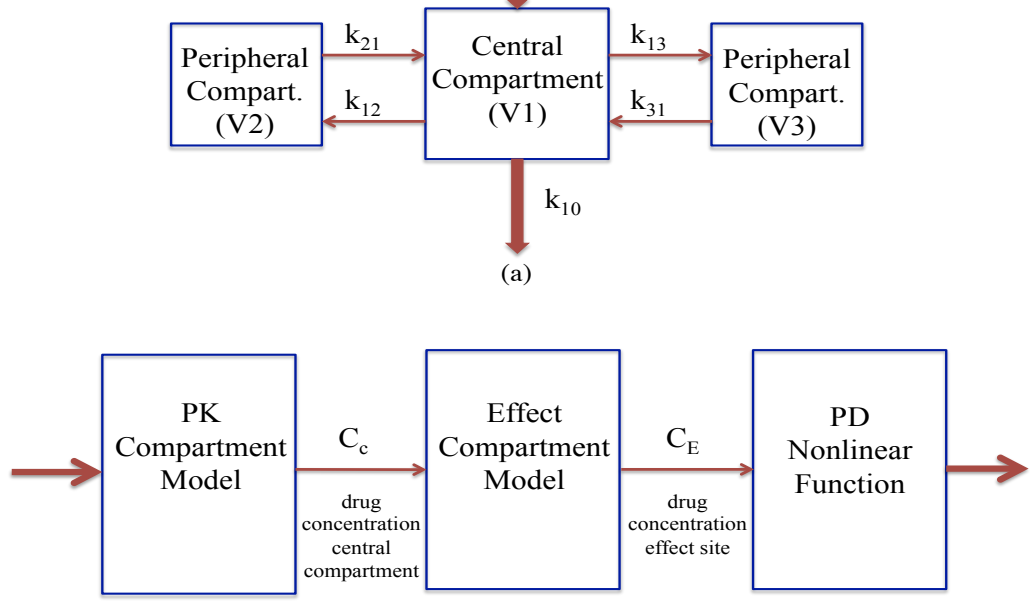

(b)

Figure 1: (a) A three-compartment pharmacokinetic model. (b) The block diagram of a combined pharmacokinetic-pharmacodynamic (PK-PD) model.

Pharmacodynamic (PD) compartment models are used to describe the relationship between drug concentration and the observed clinical effect; effect signals may be any number of patient vital signs, electroencephalogram (EEG) signals, or blood glucose levels, as examples. In this case it is frequently said that PD models address "what the drug does to the body". These models are typically given by static nonlinear functions, which are used to describe the equilibrium relationship between the drug concentration, $C$, and drug effect, $E$. A commonly used pharmacodynamic model structure is given by the well-known Hill equation,

$$
E=\frac{E_{\max } C^{\alpha}}{E C_{50}^{\alpha}+C^{\alpha}}
$$

where $E$ is the predicted effect of the drug, for example the mean arterial pressure (MAP) of the patient, $E_{\max }$ is the maximum possible effect, $C$ is the drug concentration at time $t, E C_{50}$ is the drug concentration for which $50 \%$ of the maximum effect is seen, and $\alpha$ is the Hill coefficient of sigmoidicity (a short discussion of the use of the Hill equation for PK-PD modeling follows). Frequently a non-zero baseline effect $E_{0}$ is included (i.e., effect at drug concentration 0), giving

$$
E=E_{0}+\frac{E_{\max } C^{\alpha}}{E C_{50}^{\alpha}+C^{\alpha}} .
$$

As well the effect modeled can be either positive or negative. Note that the effect being modeled can be static or dynamic; if the drug concentration changes with time the effect may change also.

Measurements of specific effects and either measured or predicted values of concentrations are used to derive the models, in accordance with the desired model structure. Concentrations at the actual effect site are required to construct accurate PD models. However, effect site concentrations are typically not available, or not easily accessed without resorting to invasive measurement techniques. Thus, predicted concentrations in the blood, derived from the PK models, are frequently used in order to capture the response from the input drug source to the gross effect output. In this case, an additional effect compartment is cascaded with the central compartment of the PK model to capture the transport time to the effect site; see Figure 1(b).

It is assumed that the amount of drug in the effect compartment itself is extremely small and has no impact on the pharmacokinetic steady-state condition. To model the time to equilibration of drug concentration at the effect site from the central compartment, an additional first order linear differential equation is used, given by

$$
\frac{d C_{\epsilon}}{d t}=K_{\epsilon 0}\left(C_{c}-C_{\epsilon}\right),
$$


where $C_{c}$ and $C_{\epsilon}$ represent concentrations in the central and effect compartments, respectively, and $K_{\epsilon 0}$ is the associated equilibration rate constant.

To determine the equilibration rate constant $K_{\epsilon 0}$ in (4), the most common approach is to use a plot of measured effect values versus blood plasma concentrations. If the time course of the effect value is considered, then a hysteresis-like response can be seen. Since blood plasma only transports the drug and is not typically the intended site of action, the resulting hysteresis loop represents the time delay from equilibrium drug concentration in the plasma to equilibrium drug concentration at the effect site. This rate constant $K_{\epsilon 0}$ can therefore be determined graphically by collapsing the hysteresis loop; parameters $E C_{50}$ and $\alpha$ may also be determined graphically [64]. Alternatively, a nested nonlinear regression algorithm to obtain optimal values of $K_{\epsilon 0}, E C_{50}$ and $\alpha$ may be used (see [26, 27] for an application to anesthesia); this is equivalent to the loop collapsing process.

\section{A short history of the Hill equation in PK-PD models}

In 1910, A.V. Hill proposed a nonlinear equation describing the relationship between two variables to describe the quantitative equilibrium relationship between oxygen tension $\left(\mathrm{pO}_{2}\right)$ and percentage saturation of hemoglobin $(H b)$ with oxygen in various salts. This equation, in a general form, is given by

$$
y=y_{\max } \frac{x^{\alpha}}{K^{\alpha}+x^{\alpha}}
$$

in the original model $y=H b_{s a t}, x=p O_{2}, y_{\max }=100$ and $K$ and $\alpha$ were parameters to be determined $[32,30]$. This model has been used since to fit experimental data from various physicochemical reactions, for example to describe enzymatic reactions, where this is frequently referred to as the Michaelis-Menten equation, and to capture dose-effect relationships in PK-PD modeling, as given by (2).

The use of the Hill equation for dose-effect modeling was first proposed by Wagner in 1968 [85], based on the logical foundation of recepter-occupancy theory. Although there is a strong connection between the Hill equation and physicochemical equilibrium reactions, when used for PK-PD modeling the parameters of the Hill equation, e.g., $\alpha, E C_{50}$ and $E_{\max }$ in (2) or (3), have little mechanistic meaning [30]. That is, these parameters do not provide much, if any, information about the physicochemical reaction involved in PK-PD processes. Nonetheless, the sigmoidal relationship expressed by (2) commonly provides a good fit to most pharmacodynamic data, in particular when the dose-effect relationship is nonlinear and saturable. Much of the success of the Hill equation in pharmacodynamic modeling is likely due to its effectiveness at fitting experimental data, and the flexibility in fitting this data resulting from the use of the parameter $\alpha$. As a result, this paradigm has become a standard in pharmacodynamics modeling.

\subsection{Compartment models in control of pharmacodynamics}

Compartment models have been used extensively in studies investigating the development of closed-loop anesthesia delivery designs, closed-loop insulin delivery designs for blood glucose control, and more recently in endocrine system studies [23]. We highlight a very short and non-exhaustive set of such studies here, focusing on anesthetics.

\subsubsection{Anesthetic pharmacodynamics}

We first note that a long and interesting history of both closed- and open-loop anesthetic delivery designs exists. As noted in Section 1 closed-loop control of anesthesia delivery has been studied for over 50 years [12], and target controlled infusion (or TCI) devices for open-loop delivery of anesthesia during surgery were first proposed over 30 years ago. For a more complete historical treatise on anesthesia, including PK-PD modeling of anesthetic agents and early control investigations, see [33]. In the following summary of prior work, we focus exclusively on the problem of controlling the level of sedation during surgery using PK-PD models.

In $[68,66,67]$, Schwilden and colleagues used median frequencies from electroencephalogram (EEG) power spectra as one measure of the sedative effects of various anesthetic agents (propofol, methohexital, 
and alfentanil) to develop PK-PD model-based adaptive feedback controllers for anesthesia delivery during both clinical studies and surgery. Gentilini and colleagues completed a number of model-based closed-loop anesthesia control studies in a series of articles [25, 24, 26, 27, 28]. In particular, in [26, 27], Gentilini et al. proposed a control scheme for the regulation of mean arterial pressure (MAP) and sedation level for the anesthetic agent isoflurane. This scheme used a cascaded internal model control (IMC) approach to regulate sedation affects of anesthesia via the bispectral index ${ }^{1}$ (BIS) level of the patient, and a threeobserver-based state feedback controller to regulate MAP. The resulting control designs were implemented in a loop-at-a-time manner, that is as nested single-input, single-output (SISO) designs. Mortier et al. also considered control of sedation level via BIS monitoring in [57], where PK-PD model-based adaptive control of propofol was proposed for surgical settings, and Morley et al. investigated the performance of PID based controllers designed based on PK-PD models to control the delivery of both intravenous anesthetics, and volatile anesthetics [56]. Soltesz, van Heusden and colleagues considered robust PID designs to control sedation level and manage pain via the delivery of proposal and remifentanil [ 76,82$]$. In an intensive care setting, adaptive and neural network based control designs for the regulation of unconciousness under administration of propofol was considered by Haddad et al. in [31].

Other aspects of anesthesia control include management of analgesia and neuromuscular blockades. The effects of neuromuscular blockade agents are typically uncoupled from sedative and analgesic agents, and can thus be viewed as a separate control problem. At present, there are no accepted sensing devices and/or quantified human response signals to indicate adequate analgesia; many commonly used analgesics (opioids, for example) present synergistic effects with sedative agents. This presents a viable direction of continued study for the application multivariable control methods.

\subsubsection{Insulin pharmacodynamics}

With the increasing availability of subcutaneous insulin delivery pumps and subcutaneous continuous blood glucose monitors, closed-loop control of insulin and glucose levels in patients with diabetes has become a viable short-term goal. Compartment models have been used extensively for PID control and MPC designs over the history of investigations into closed-loop control of blood glucose levels; we note a few recent highlights of this work here. In [61], mass balancing techniques, focusing on tissues important to glucose or insulin dynamics, are used to derive nonlinear compartment models for diabetic patients. Compartments representing the heart/lungs, liver, kidney, brain and gut, and a periphery compartment, are considered, incorporating transport resistance (i.e., time delay) effects where relevant. The output to be controlled is the arterial glucose concentration, which is regulated by the insulin infusion rate. An identification algorithm is introduced to derive a linear approximation to the nonlinear compartment model, and subsequent linear MPC designs are developed and evaluated in simulation studies. In [9], a simple compartment model relating glucose infusion to glucose concentration is used to evaluate a glucose clamp algorithm, which is shown to be equivalent to PI control with time-delay compensation; this control algorithm is aimed at reaching a specific blood glucose concentration set point after a fixed number of sample times in the presence of meter bias and noise. The evaluation of plasma insulin and glucagon ${ }^{2}$ levels in determining whether microdosing of glucagon is effective at preventing hypoglycemic events is considered by El-Khatib, et. al in [22, 63], where PK models of the absorption and clearance of insulin are used in MPC designs that control insulin dosing, and independently, customized PD designs are used to control glucagon dosing, giving a loop-at-a-time multi-input multi-output (MIMO) design.

\subsubsection{Challenges}

Although a large body of work has resulted from the use of PK-PD models for control of pharmacological agents, there are notable challenges presented by this modeling paradigm. The mathematical models resulting

\footnotetext{
${ }^{1}$ The BIS measure is a statistical index, based on phase and frequency relations between the component frequencies in EEG recordings, which found widespread acceptance over the past two decades as a means of sensing and quantifying the depth of sedation $[75,29,69,70,58]$.

${ }^{2}$ Glucagon is a hormone produced by the pancreas, which increases the concentration of glucose in the blood, thus having an opposite effect to that of insulin.
} 
from the use of the PK-PD paradigm are inherently single-output (one effect is considered per model), and most commonly single input (although there has been a recent effort to derive combined therapy models, that is, PK models with two drug inputs [55, 15, 40, 52]). As a result, these models are incapable of capturing the response to disturbances, for example heightened activity levels in the case of modeling blood glucose levels, or extensive blood loss in the case of modeling anesthetic response in surgical settings; the inevitable synergies occurring with multi-drug therapies, which is particularly common with sedative and analgesic anesthetic agents; and relationships among different effects or vital signs in the human body. Further, depending to some extent on the identified value of the parameter $\alpha$, these models can be highly nonlinear, and as noted just following equation (1), pharmacokinetics models represent non-negative systems, which lead to potential constraints on the control design process. Perhaps most importantly, there are no direct mechanisms in this model structure for capturing general inter-patient dynamics, intra-patient variability or model uncertainty.

A few alternatives have been proposed to the standard compartment models for capturing pharmacodynamic behavior. In [81], personalized models of blood glucose concentrations resulting from insulin injections are developed using frequency domain estimation methods. Specifically, third-order parametric models are identified using an output-error structure, which capture the individual dynamics around expected bandwidths; these models are intended to be control relevant, i.e., useful for the purpose of glucose control rather than aimed at minimizing prediction errors. Robustness of resulting closed-loop control designs based on these individualized models is confirmed using simulations. Similar models are augmented and considered in an evaluation of LPV control methods aimed at minimizing risks of hypoglycemia in [14]. Piecewise-linear and LPV MIMO models have been proposed for capturing patient response to anesthetic agents, surgical stimuli and disturbances in $[50,49]$. These models have demonstrated good predictive capabilities, but have not yet demonstrated particularly strong suitability for control design. Linear data-based state-space models, derived over restricted ranges of system operation, are also considered for the purpose of applying adaptive control techniques [42, 43]; further details on model construction, closed-loop control designs and related simulation results for these alternative approaches to the anesthesia control problem are presented in Sections 3 and 4.

\section{MIMO Modeling for Control of Anesthesia Delivery}

Closed-loop delivery of anesthetics can produce a number of benefits, such as reducing the overall amount of anesthetics required for individuals, and allowing the anesthesiologist to focus on safety critical issues arising due to the surgical demands on the patient. The primary advantages resulting from closed-loop anesthesia delivery would include reduced recovery times and improved long-term patient outcomes $[1,45,38]$, and reduced pharmaceutical costs.

There have been numerous closed-loop anesthesia studies to date, some of which were noted in the preceding section. These studies almost exclusively have used SISO models and SISO control design approaches. However, control of anesthesia in a surgical setting is undeniably a MIMO problem. The anesthesiologist is responsible for adjusting the delivery of multiple anesthetic agents given to the patient, aimed at maintaining adequate and appropriate levels of hypnosis, or lack of consciousness; analgesia, or lack of pain perception and the resulting autonomous system effects (e.g., increased heart rate and blood pressure); and muscle relaxation or lack of movement. At the same time, the anesthesiologist must maintain the patient's ventilation parameters and monitor all cardiovascular and respiratory functions, including, but not limited to, heart rate $(\mathrm{HR})$, blood pressure, oxygen saturation and exhaled carbon dioxide $\left(\mathrm{CO}_{2}\right)$ levels. Invasive montitoring is also sometimes used by anesthesiologists during surgery to monitor arterial blood pressure, right- and left-heart filling pressures, and pulmonary arterial pressures. Cardiac output (CO) signals may also be monitored, for example by thermodilation methods, and then used to derive systemic vascular resistance, pulmonary vascular resistance and a number of other cardiac performance measures. Additionally, gas concentrations, blood-sugar levels, electrolyte concentrations and coagulation parameters may be observed by the anesthesiologist using intra-operative blood samples. The anesthesiologist combines the information provided by any or all of these patient indicators, with his or her knowledge and experience of individual drug dose-response effects and the synergistic effects of various drug combinations, to determine if and when 
the patient is properly anesthetized.

Currently, there does not exist a single well-accepted indicator for anesthetic adequacy, and in fact it is obvious that a single indicator will not suffice for describing adequate levels of the three main components of anesthesia. The electroencephalogram (EEG) may be monitored as one measure of the depth of sedation, or hypnosis, however, difficulties have been encountered in quantifying hypnotic depth in terms of EEG measurements, including varying effects on EEG by different anesthetic agents [77]. The BIS level was introduced in the mid 90's as an alternative measure of sedation level to raw EEG signals; recently there has been some controversy associated with this index [46], however the National Institute for Health and Care Excellence continues to recommend its use during application of intravenous anesthesia.

In the following we review our work on modeling and control of anesthetic pharmacodynamics, outlining the MIMO piecewise-linear and LPV modeling approaches we have considered. Simulation results and comparisons from these modeling efforts are provided. In Section 4, we provide an overview of control design results including LPV techniques, and $\mathcal{L}_{1}$-adaptive methods. Some of these results have been presented previously in $[50,5,62,41]$. Our prior modeling and control studies have been based on data collected from a clinical study of 10 volunteers, which we have used as a comparative case study for evaluating different modeling and control paradigms.

\section{Clinical Trial Data}

The original study from which our data was obtained was designed to define the relation between clinical evaluation of the state of consciousness, explicit recall, drug concentrations and BIS effects of the anesthetic agent isoflurane when administered to healthy volunteers (referred to as patients henceforth) under controlled conditions. A series of external stimuli were also applied to the patients throughout the trial. These input stimuli included: laryngeal mask insertion and removal, performed when the patient was considered completely sedated; evoked potential evaluations involving the application of short electrical stimulation signals to the wrist of the patients at a period of every 3 seconds and up to $100 \mu \mathrm{A}$ and $100 \mathrm{~V}$ amplitude; and alertness evaluations including yelling at, shaking, and squeezing the trapezius muscle of the patient. (See [50] for further details and references regarding the clinical protocols). Quantitative models of the stimuli applied to the patients during the study were developed as part of our early modeling studies [50]. Previous to this, quantitative models for similar stimuli inputs in clinical trials had been adopted by Spreckelsen and Bromm [84], by Derighetti, et al. [17] and by Frei, et al. [24].

Time-synchronized output measurements included BIS levels, MAP and HR. BIS values range from 0-100, where a BIS value near 100 corresponds to a completely alert state, a BIS value around 60 corresponds to a moderate hypnotic state, a BIS value around 40 corresponds to a deep hypnotic state, and very low BIS values are referred to as characterizing a profound anesthetic state $[35,70]$. For healthy individuals, normal ranges for MAP are between 70 and $110 \mathrm{mmHg}$, and the average resting HR for normal adults is around 70 beats per minute [11].

An example of a set of data taken from one subject is shown in Figures 2 and 3. Note that the maximum amplitude of the external stimuli has been normalized to one, with relative weightings determined implicitly by the identification algorithms. Also note the distinct transitions, or the effective switching that has been attained between the BIS levels in the plot presented in Figure 3.

\subsection{Multivariable Models}

It was observed that the responses of the subjects in the clinical trial data transitioned from one set of linear dynamic behaviors to another as the course of anesthesia took the subjects from alert to sedated conditions. Based on this, we evaluated the use of MISO and MIMO switching system models where the underlying subsystems are linear state-space models over which the subjects' responses switch based on their sedative state. Piecewise-linear (PWL) and LPV models were both evaluated. We summarize the results of those modeling studies here. 

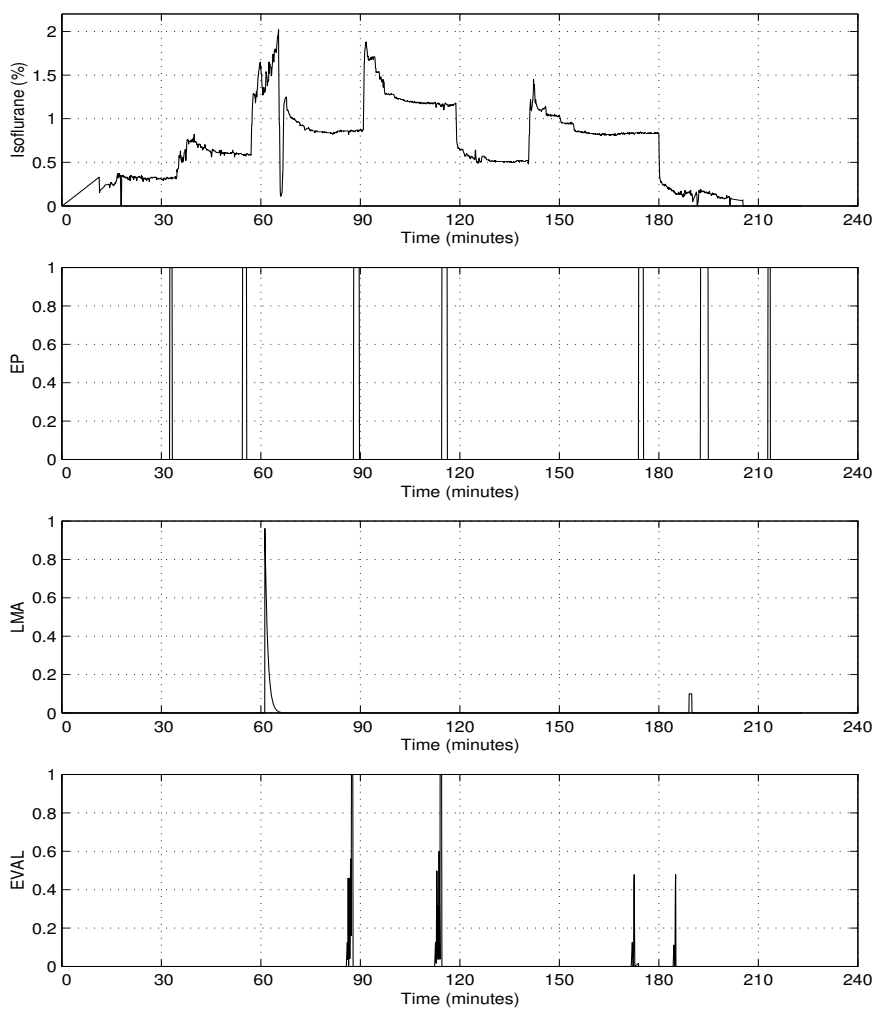

Figure 2: Isoflurane and stimuli inputs versus time (patient 1): the plot with vertical axis labeled EP represents evoked potentials stimuli, that labeled LMA represents the laryngeal mask insertion process, and that labeled EVAL represent the alertness evaluation tests. 

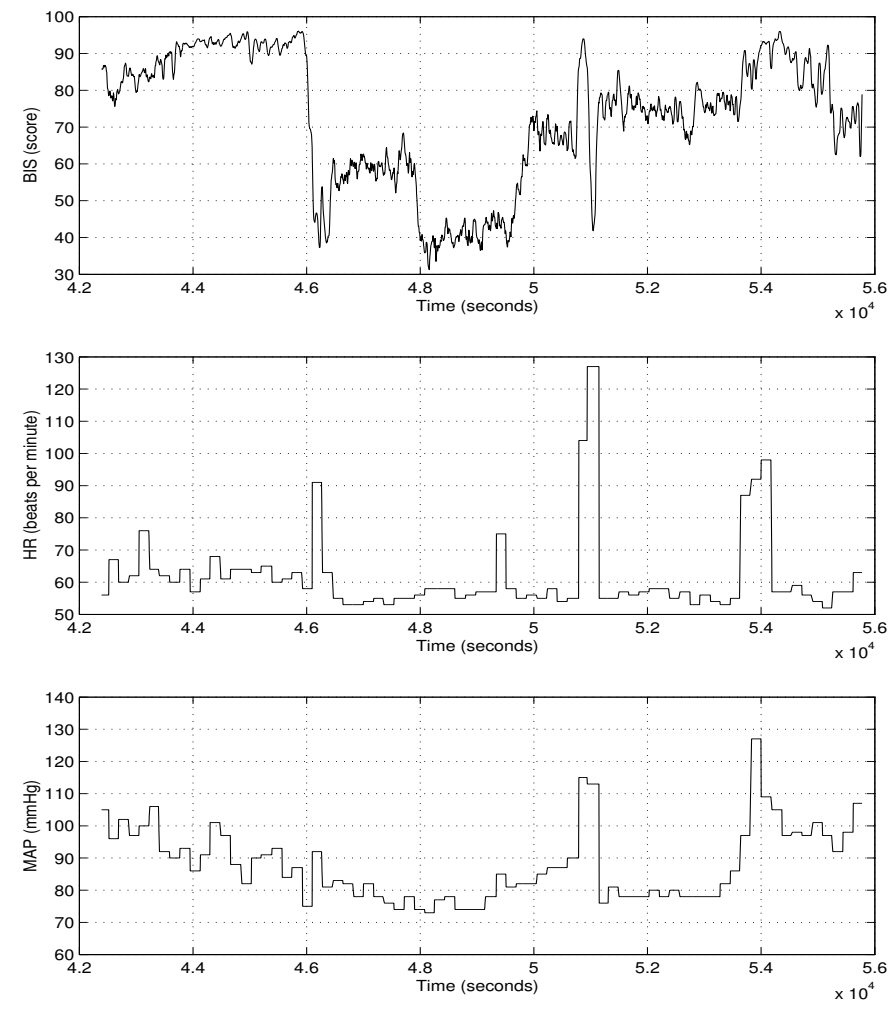

Figure 3: BIS, HR and MAP outputs versus time (patient 1). The horizontal axis represents clock time, with the trial start time representing approximately 11:20 AM. 


\subsubsection{Piecewise-linear Models}

Both PWL and LPV models effectively captured the sigmoidal dose-effect response typically seen with anesthetics. Constituent subsystems in the PWL models we proposed were constructed using subspace identification methods applied to the clinical trial data (see, for example [51, 83], and the references therein for a subspace identification overview). Two linear state-space subsystems were identified, denoted

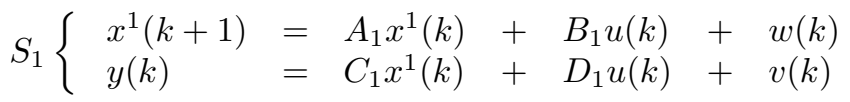

and

$$
S_{2} \begin{cases}x^{2}(k+1) & =A_{2} x^{2}(k)+B_{2} u(k)+w(k) \\ y(k) & =C_{2} x^{2}(k)+D_{2} u(k)+v(k)\end{cases}
$$

which model the patient response in the awake and sedated states, respectively. Here $u(k)$ and $y(k)$ represent the sampled input and output data (e.g., isoflurane and stimuli inputs, and BIS and vital sign outputs), $x(k)$ is the state vector, $w(k)$ and $v(k)$ are assumed to be white-noise processes, and $A_{i}, B_{i}, C_{i}$, and $D_{i}, i=1,2$, are constant real-valued matrices estimated by the subspace identification process.

Measured BIS values were used to determine which of the two subsystem models of a patient's response to anesthesia and stimuli was appropriate: the alert condition or sedated condition model (the latter included both moderate and deeply sedated conditions). Switching over these two model choices occurs at a BIS threshold value of 70 (it has been noted that approximately $50 \%$ of the population will be unconscious at a BIS value of $70[70]$ ). Upon switching from one subsystem, $S_{j}$, to the other subsystem, $S_{i}$ the initial state for the subsystem $S_{i}$ was calculated directly from the most recent output of $S_{j}$ (note the outputs contained no feedforward information from the inputs, thus $D_{1}$ and $D_{2}$ are both zero matrices). Although the output remains continuous with this switching model, a jump in state values may result at the switching instant. One example of the partitioning of data based on a BIS level of 70 can be seen in Figure 4. Simulations based on the resulting set of models are presented in Section 3.2.

\subsubsection{LPV Models}

Most PWL synthesis strategies are based directly on knowing the partitions or cells in the state space, and the respective transitions between these partitions, which requires direct knowledge (or estimation) of both the current state and the partitions of the state space. This information is not available from models constructed using black-box identification methods. To address this issue, we considered linear parametervarying models and control methods to complete our original closed-loop designs. In the framework we considered, the patient's BIS level was viewed as a measurable time-varying system parameter over which the system dynamics vary (smoothly), thus we have quasi-LPV models. (See [73] for a clear overview of the LPV framework). One benefit of using LPV approaches is that these models are able to capture the transition from alert to sedate and back in a smooth manner. To transform our PWL models to LPV models, we applied a simple curve fitting process using the system realizations obtained for the patients in the awake and sedated states, $S_{1}=\left\{A_{1}, B_{1}, C_{1}, 0\right\}$ and $S_{2}=\left\{A_{2}, B_{2}, C_{2}, 0\right\}$ respectively. We defined the relationships for the multi-input to BIS output models by

$$
P_{B I S}\left\{\begin{array}{l}
A(\delta)=\frac{\delta(\delta-1)}{2} A_{1}+\frac{\delta+1}{2} A_{2} \\
B(\delta)=\frac{\delta(\delta-1)}{2} B_{1}+\frac{\delta+1}{2} B_{2} \\
C(\delta)=\frac{\delta(\delta-1)}{2} C_{1}+\frac{\delta+1}{2} C_{2},
\end{array}\right.
$$

where the value of $\delta$ at any time $k$ is based on patient measured BIS values, and is given by

$$
\delta(k)=1-\frac{2}{1+\exp ^{\eta *(70-B I S(k))}} .
$$

The determination of the function $\delta(k)$ is consistent with the definition for BIS levels, namely that a BIS value around 100 corresponds to a completely alert state and a BIS value around 40 corresponds to a deep 

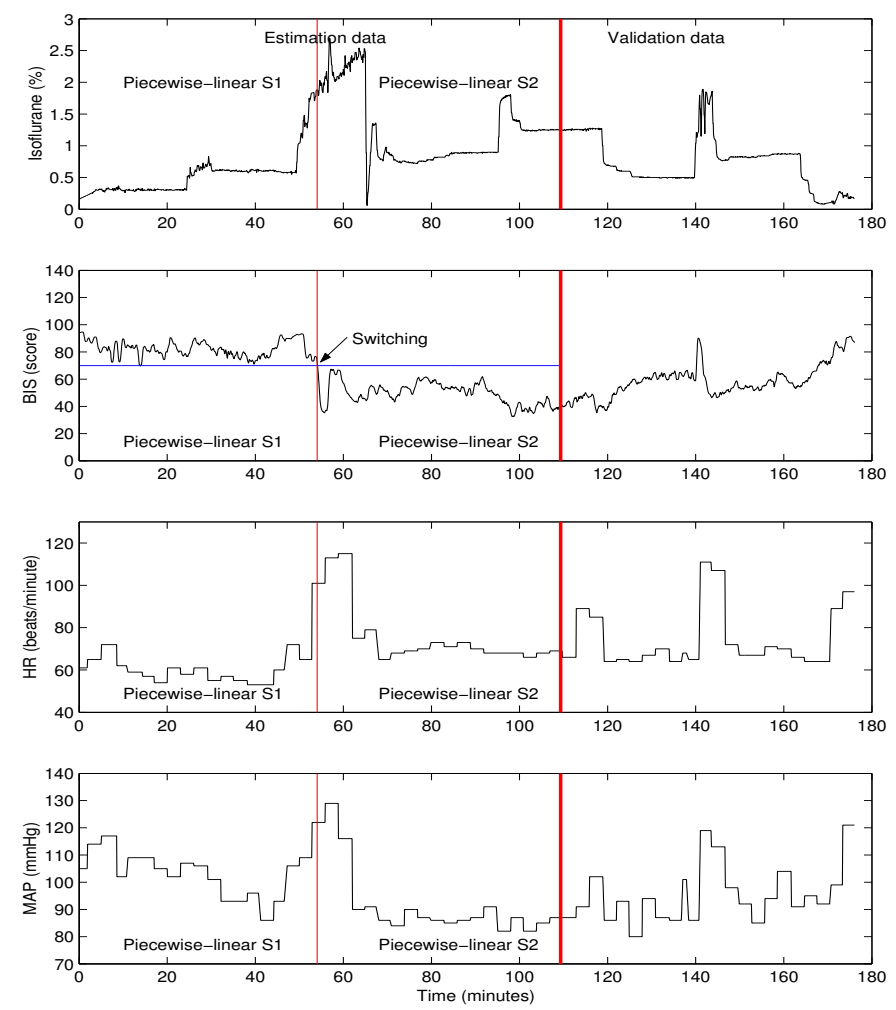

Figure 4: Partitioning of input and output data for piecewise-linear modeling (patient 3). The horizontal axis represents elapsed time during the clinical trial. 
hypnotic state. As a result, $\delta=-1$ represents patients being in a totally awake state and $\delta=1$ represents patients being in a deeply sedated state. We set the slope $\eta=0.2$ to provide a smooth piecewise-linear response.

For the multi-input to MAP/HR output models, the LPV models were determined by the relationships

$$
P_{M A P / H R}\left\{\begin{array}{l}
A(\delta)=\frac{1-\delta}{2} A_{1}+\frac{1+\delta}{2} A_{2} \\
B(\delta)=\frac{1-\delta}{2} B_{1}+\frac{1+\delta}{2} B_{2} \\
C(\delta)=\frac{1-\delta}{2} C_{1}+\frac{1+\delta}{2} C_{2} .
\end{array}\right.
$$

The resulting LPV models had unnecessarily high dimensions, for example a typical patient model had dimensions $n_{1}=36$ in the $\delta$ parameter and $n_{2}=4$ in the state. Multidimensional model reduction methods were applied to the LPV models, leading to reduced dimension models of order $n_{r_{1}}=15$ in the $\delta$ parameter and $n_{r_{2}}=4$ in the state. Model reduction errors were approximately $5 \%$ or less (based on a cumulative generalized Hankel singular value criteria; see [4] for a discussion of reduction methods and algorithms).

\subsubsection{PK-PD models from the clinical trial data}

For comparison, the dosing and related effects of isoflurane were also modeled using traditional PK-PD models. To construct the PK model, the mammillary compartment model identified by Yasuda, et al [86] was used.

The Yasuda model is given by the equations

$$
\begin{aligned}
\dot{x_{1}} & =I+x_{2} k_{21}+x_{3} k_{31}+x_{4} k_{41}+x_{5} k_{51}-x_{1} k_{10}-x_{1} k_{12}-x_{1} k_{13}-x_{1} k_{14}-x_{1} k_{15} \\
\dot{x_{2}} & =x_{1} k_{12}-x_{2} k_{21}-x_{2} k_{20} \\
\dot{x_{3}} & =x_{1} k_{13}-x_{3} k_{31} \\
\dot{x_{4}} & =x_{1} k_{14}-x_{4} k_{41} \\
\dot{x_{5}} & =x_{1} k_{15}-x_{5} k_{51}
\end{aligned}
$$

where $I$ is the inspired concentration, $x_{i}$ is the amount of drug in the $i^{\text {th }}$ compartment, $k_{i j}$ is the transfer rate constant from the $i^{t h}$ compartment to the $j^{t h}$ compartment, and $k_{10}$ and $k_{20}$ are the drug elimination or clearance rate for the first and the second compartments, respectively. Numerical values for the constants $k_{i j}$ and $k_{i 0}$ were determined empirically. As isoflurane is a volatile anesthetic, the central, or first compartment represents the lungs. One benefit of using volatile anesthetics is that exhaled or end-tidal gas concentrations, which are easily measured, correlate well with anesthetic partial pressure at the intended site of action for the healthy volunteers; this allows for a direct validation of the model with the data. The second compartment represents the vessel-rich group (including highly perfused organs such as the brain, heart and liver), and the third compartment represents muscle tissue. The fourth and fifth compartments represent fatty tissues, with the fourth compartment used to represent the layer of fat receiving anesthesia from adjacent (vesselrich) organs via inter-tissue diffusion. In this model elimination of anesthetics occurs at two sites, the central compartment and the second compartment, with the elimination mechanisms being exhalation, and metabolism occurring via the liver. A comparison of predicted results from the Yasuda model versus measured responses for all patients in our data set yielded an average MSE of \%3.0.

To construct the PD models, the only input used from the data was isoflurane, the anesthetic. In this case, the PD models were individualized and not mean-based (as is the Yasuda PK model), that is, one PD model was constructed for each patient for each effect. The isoflurane to BIS dose-effect relationship was modeled by the function

$$
B I S=B I S_{0}\left(1-\frac{C^{\alpha}}{E C_{50}^{\alpha}+C^{\alpha}}\right),
$$

where $B I S_{0}$ represents the base, or no-drug, patient BIS value (maximum of 100). To compute the parameters $E C_{50}$ and $\alpha$, a nested two stage nonlinear optimization algorithm was used, based on the nonlinear regression algorithm proposed by Genitilini, et. al, in [27, 26]. Estimated values of $K_{\epsilon 0}, \alpha$ and $E C_{50}$ forming the BIS compartment models for all patients were determined; the parameters computed were comparable 
to those found in [27] and [58], given the protocols of the respective studies. A more detailed discussion is given in [50].

Construction of PD models of the MAP and HR responses to isoflurane were also attempted. The optimal $K_{\epsilon 0}$ value in the first stage should be determined graphically by "collapsing" the hysteresis loop, as mentioned in Section 2. However, using the clinical data available to us a complete hysteresis loop could not be generated for HR and MAP effects, and this parameter could not be computed. We also note that prior studies indicated that the overall trend of the heart rate response to isoflurane is not consistent, nor is it clearly understood (see $[24,20,16,21]$ ). For these reasons, we did not consider these results directly in our comparative analysis, although we do include some of these simulation results herein.

\subsection{Model Simulation Results}

SISO piecewise-linear BIS response models (with only the isoflurane input), multi-input piecewise-linear BIS response models (with isoflurane and external stimuli inputs), and MIMO piecewise-linear models capturing BIS, MAP and HR responses were constructed and their respective simulated responses compared to standard SISO PK-PD models. Examples of measured and predicted outputs for piecewise-linear MIMO response models and the PK-PD response models are shown in Figures 5 and 6 . The point at which the data record was separated into data subsets for estimation and validation of the piecewise-linear models is indicated by the dashed vertical line in the plots. Note that the entire data record is needed to construct PK-PD models. A comparison of the PK-PD and the piecewise-linear models was completed using (1) predictive capabilities, (2) error signal means and variances over pooled data, (3) normalized errors for individual patient data sets, and (4) computational effort. Based on direct observation of prediction/estimation capabilities, in general the piecewise-linear model responses compared favorably to the PK-PD model responses (see [48] for a complete set of simulation results).
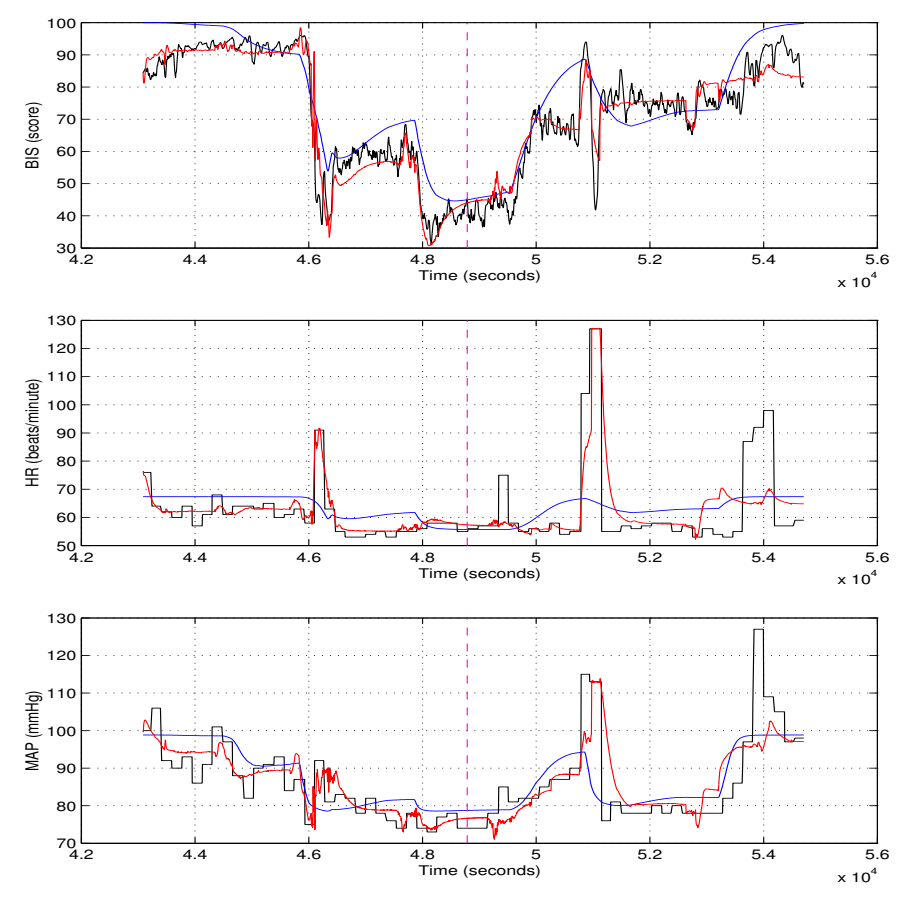

Figure 5: Clinical data (black), simulated PWL responses (red) and simulated PK-PD responses (blue) for Patient 1.

We further found that the piecewise-linear models for one individual provided reasonable central models, i.e., models applicable to a group of subjects, in that simulated output responses obtained using the input data set for one patient (e.g., patient 1) and the piecewise-linear model for another patient (e.g., patient 3) 

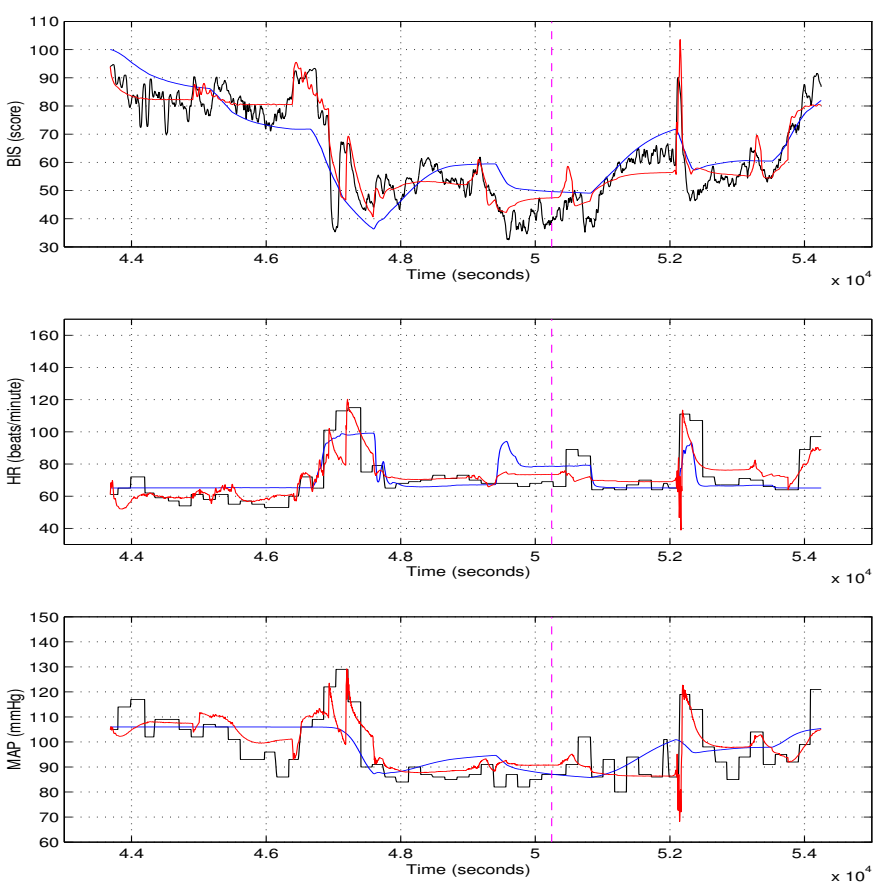

Figure 6: Clinical data (black), simulated PWL responses (red) and simulated PK-PD responses (blue) for Patient 3.

produced an acceptable fit to the output data for the original patient. For one example see Figure 7; an additional example is given in Figure 8. An evaluation of all combinations of BIS piecewise-linear models and data sets were completed. These evaluations indicated that the models for patients $1,6,7$ and 8 provide adequate fits to all other data sets, and the models for patients 2 and 5 provide adequate fits for a subset of the remaining data sets. This leads to the question of whether central models exist for specific segments of the population, that is, to what extent individual covariates such as age, gender, body weight, ethnicity and/or coloration [47] are correlated with how well models based on one patient's data set predict the responses exhibited by another patient's data sets.

Quantitative comparisons of the results from the PK-PD and both the SISO and MISO piecewise-linear modeling efforts were completed; error means and variances were evaluated. The mean over individual data sets of the error signal $e(t)=y(t)-\hat{y}(t)$ was computed, and population means over all data sets were compared using a $t$-test $(p<0.05)$. Using the full data set to estimate the PK-PD model parameters, values for the population mean and variance of the error signal were also obtained. Comparing the PK-PD error mean and variance values to those from the MISO piecewise-linear models, an $F$-test indicated no significant difference between the two population variances ( $F$ test: $p<0.05)$. However, the $t$-test indicated the mean error from the PK-PD models was significantly larger than the mean error from the piecewise-linear models (one-tailed paired $t$ test: $p=0.011$ ). Summarizing, in both the SISO and MISO cases, the piecewise-linear models proposed outperformed the PK-PD models by this measure. The average normalized error for the piecewise-linear models was 0.0944, and for the PK-PD models the average normalized error was 0.1341; recall the entire data set was required to estimate the PK-PD model parameters. Computational efficiency was also evaluated for the PK-PD and piecewise-linear approaches described. In brief, the PD models required a factor of 5 to 20 times more computation time than did the piecewise-linear models (see [50] for more details).

LPV model responses were similar to their associated PWL model responses. Reduced LPV models were then computed for use in control synthesis in place of the original high dimension LPV models; one example of a simulated response for a full versus a reduced LPV model is given in Figure 9.

Using the LPV models and sedated portions of the identified PWL models, robust and adaptive controllers 

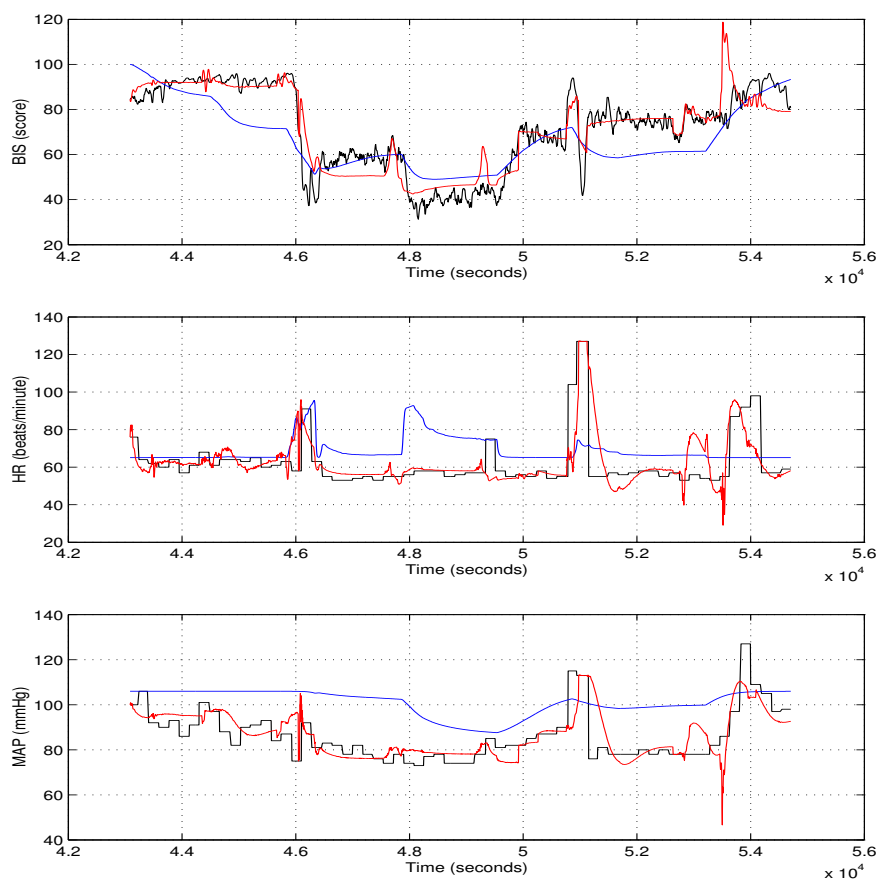

Figure 7: Clinical data (black line), PWL simulated responses (red line), and PK-PD simulated responses (blue lines), for Patient 1 data using Patient 3 models.
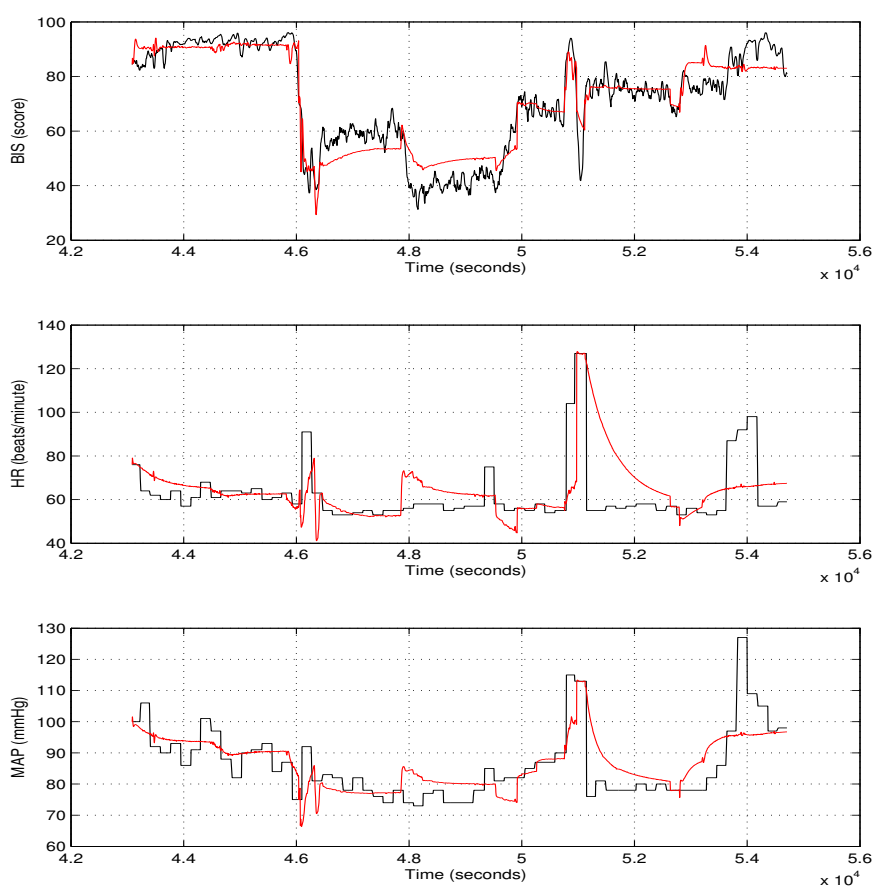

Figure 8: Clinical data (black lines) and simulated responses (red lines) for Patient 1 data using Patient 8 piecewise-linear model. 

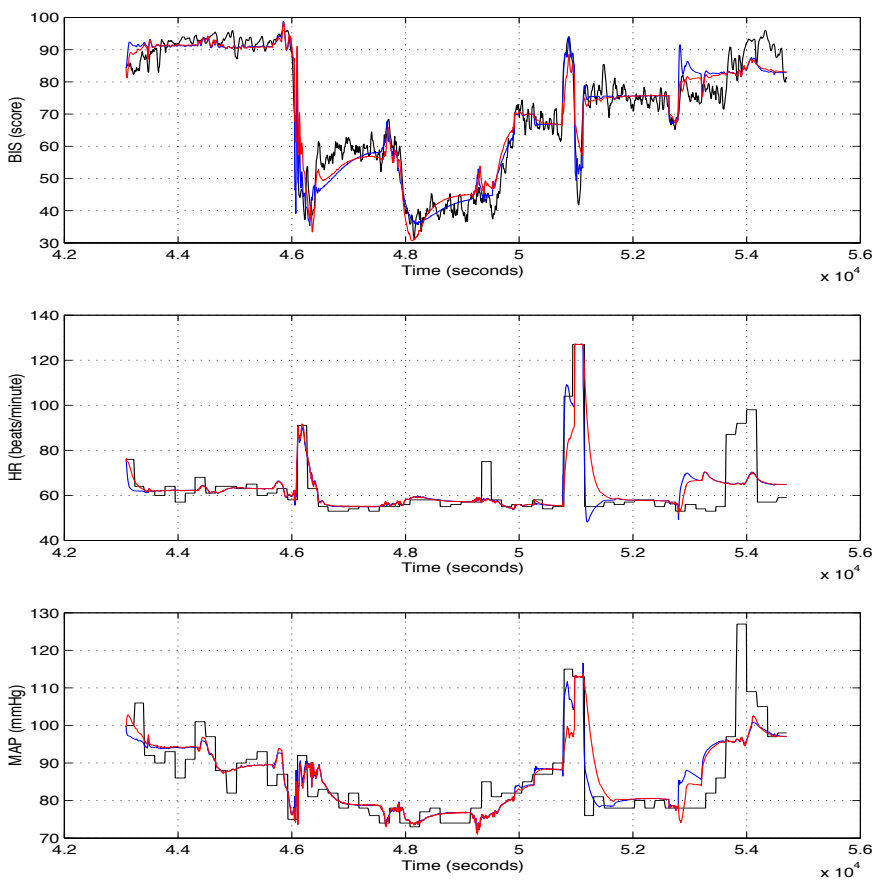

Figure 9: Clinical data (black lines), full dimension LPV response (red line) and reduced dimension LPV response (blue line).

were designed and implemented in simulations. A discussion of these results follow.

\section{Feedback Control Methods for Anesthesia Delivery}

We considered two control synthesis approaches, both amenable to MIMO models, namely LPV, or robust gain-scheduling methods, and $\mathcal{L}_{1}$-adaptive methods. Prior to presenting a sample of those results, we overview the basic control objectives.

\section{Control Objectives}

The main control goals are to track the BIS reference signal, attenuate the effect of disturbances, and for safety reasons, maintain the HR and MAP within given "safe" ranges. At the same time, the overall consumption of anesthetics should be minimized. Although these goals lead to a multi-objective control problem, in the initial designs we focused primarily on tracking of the BIS reference trajectory when subjected to external disturbances (i.e., external stimuli), with maintenance of desired MAP ranges as a secondary goal. Desired reference trajectories of patient BIS values for all data sets were determined by the attending anesthesiologist.

Our original objectives considered control through the complete anesthesia cycle: from the alert state to induction of sedation, through clinical trial stimuli, and back to the alert state after stopping delivery of anesthetics. LPV control synthesis algorithms such as those developed in $[74,72,7,60,6,3]$ were designed for this complete cycle and implemented in simulation studies. For actual physical implementation of closedloop control, it was determined that the attending physician will perform the initial induction from the alert state to the lightly-sedated state in order to closely monitor initial patient response. Upon being lightly sedated and observed for safety reasons, the patient then will be switched to the automated control design by the anesthesiologist. In this case, the control design is intended only for use during the sedated state. We designed and implemented robust adaptive controllers using the $\mathcal{L}_{1}$-adaptive methods proposed in [13] for this latter setting. In the sequel we provide a brief overview of preliminary control design results. 


\subsection{Linear Parameter-Varying Control Results}

The LPV control synthesis approach was developed in the 90's based on the small-gain theorem [87]. In this approach to control design, state-space entries of both plant and controller are assumed to be linear fractional functions of time-varying parameters over which the system dynamics evolve. The stability of the closed-loop system is guaranteed using constant quadratic Lyapunov functions. For details and further references, see $[60,3,7,18]$.

The general LPV control design structure is shown in Figure 10. For our problem, the upper two blocks in this figure, denoted $M$ and $\Theta$, would represent the system, i.e., the patient, whose dynamics evolve with respect to the time-varying parameter denoted by $\delta(k)$, as well as time, that is, with state equations

$$
\begin{aligned}
& x(k+1)=A(\delta(k)) x(k)+B(\delta(k))\left[\begin{array}{l}
w(k) \\
u(k)
\end{array}\right] \\
& {\left[\begin{array}{l}
z(k) \\
y(k)
\end{array}\right]=C(\delta(k)) x(k)+D(\delta(k))\left[\begin{array}{l}
w(t) \\
u(t)
\end{array}\right] .}
\end{aligned}
$$

Here $M$ is a constant matrix determined by $A(\delta), B(\delta), C(\delta), D(\delta)$, the matrix-valued functions defined as in (5) and (7), $\Theta(k)=\left(\delta(k) I_{n_{1}}, q I_{n_{2}}\right)$ with $q$ representing the shift operator, $u$ represents controlled inputs (e.g., Isoflurane) and $w$ the disturbances, and the outputs $z$ and $y$ are the MAP and BIS signals. The lower two blocks in this figure, denoted $K$ and $\Theta_{K}$, represent an $\mathcal{H}_{\infty}$ controller designed to satisfy stability and performance specifications over the range of values taken by $\Theta$. LPV controllers have dynamics that also evolve with respect to the system time-varying parameters, in this case $\delta(k)$.

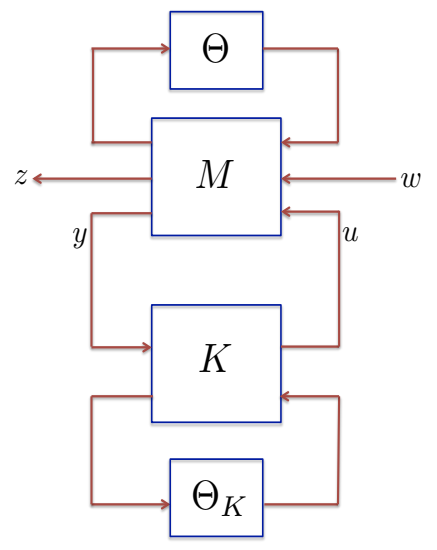

Figure 10: The general LPV control structure: the upper blocks represent the parameter varying plant, and the lower blocks represent the controller which is scheduled over the same parameter set.

LPV controllers were computed for each individual data set by solving the associated linear matrix inequality problem (as given, for example, by theorem 11.5 in [18]). Figure 11 shows one example of an LPV control simulation result for BIS reference input tracking; a second example is given in Figure 12. Normalized tracking error tabulations were computed based on $\frac{\|\hat{y}-r e f\|_{2}}{\|r e f\|_{2}}$, where ref represents the desired BIS reference trajectory and $\hat{y}$ represents the simulated control output. The average normalized tracking error for the original set of LPV control simulations was approximately $15 \%$.

Controller cross-validations amongst patients for the BIS reference tracking were evaluated, also. Controllers generated from the estimated model for one subject were used to close the loop with other subjects' data and associated models. An example is shown in Figure 13. A number of such combinations were evaluated. The results indicated that the controller generated for patient 1 when used as a controller for patients 2 and 6 performed similarly to the individually designed controllers for those patients; similarly the controller generated for patient 3 when used as a controller for patients 5, 7, and 8 lead to little degradation 

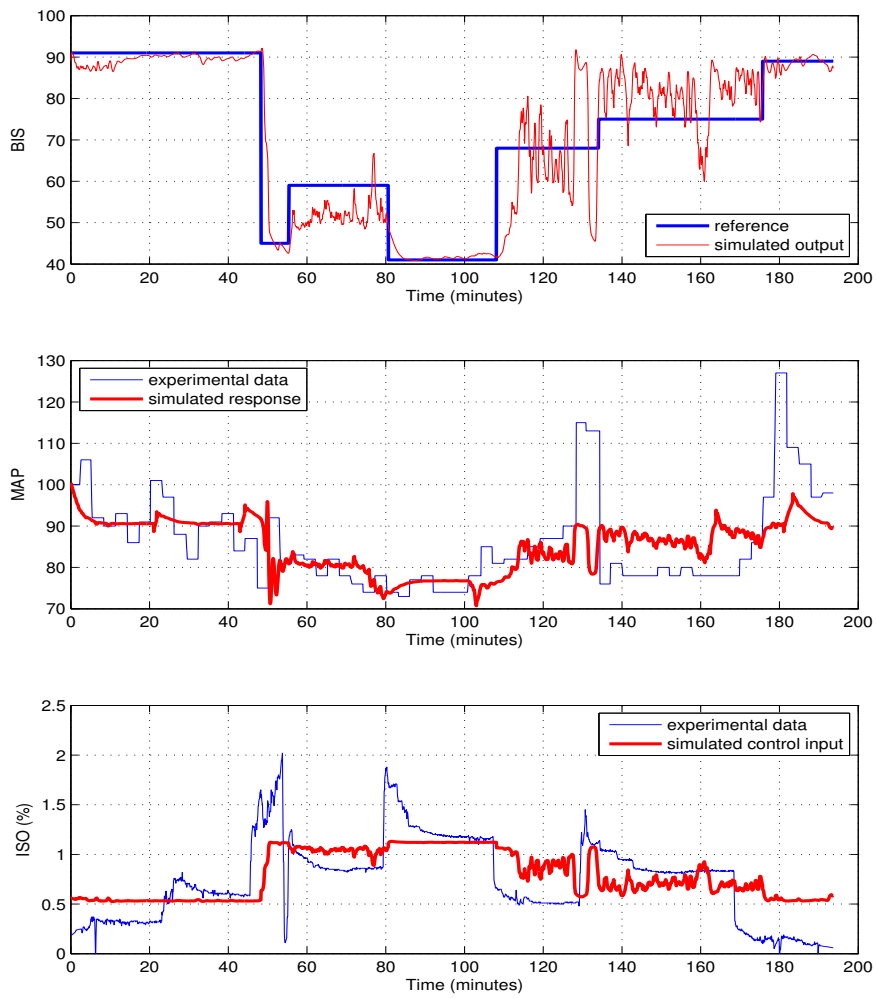

Figure 11: Control simulation result of BIS reference tracking and MAP range for patient 1: In this 3by-1 plot, the upper plot shows the desired BIS reference trajectory (blue line) and the control simulation output (red line); the center plot shows the MAP data (blue line) and the control simulation output (red line); the lower plot represents the isoflurane control input (red line) (in \%volume concentration) and the isoflurane measurements from the associated clinical trial (blue line). This BIS reference trajectory attempts to maintain the simulated patient response just below and just above the BIS switching threshold of 70 in the latter half of the simulation, a demanding test for the controller.

in performance. This indicates some potential for the design of nominal models for population subgroups (based for example on covariate analyses) combined with robust and/or adaptive control techniques.

We now summarize the initial implementation results from the $\mathcal{L}_{1}$-adaptive control designs.

\section{2 $\mathcal{L}_{1}$-Adaptive Control for Anesthetic Pharmacodynamics}

The $\mathcal{L}_{1}$-adaptive control architecture was first proposed by Cao and Hovakimyan in [13]. This method is explicitly intended for use in settings where the system output must follow a given reference signal, but in which it is expected that there are noticeable modeling uncertainties and disturbances which the controller must counteract. The $\mathcal{L}_{1}$-adaptive control architecture is meant to provide asymptotic tracking with uniformly bounded inputs and outputs. The basic structure of the $\mathcal{L}_{1}$-adaptive controller incorporates a predictor, a parameter adaptation law, and naturally a feedback control law. We present a simplified discussion of the $\mathcal{L}_{1}$ design process here, and then present the results of using these methods in the anesthesia (case study) control problem. An early version of applications of $\mathcal{L}_{1}$ methods to the anesthesia problem may be found in [62].

First, we note that $\mathcal{L}_{1}$-adaptive control design is based on the use of standard LTI transfer function models, i.e., we assume we have a system modeled as $y(s)=G(s)(u(s)+d(s))$, where in our case $y(s)$ would represent the BIS and/or MAP output, $u(s)$ would represent the anesthesia input, $d(s)$ would represent the disturbances, and $G(s)=C(s I-A)^{-1} B$ is a simple model for the system; we note that there are some 

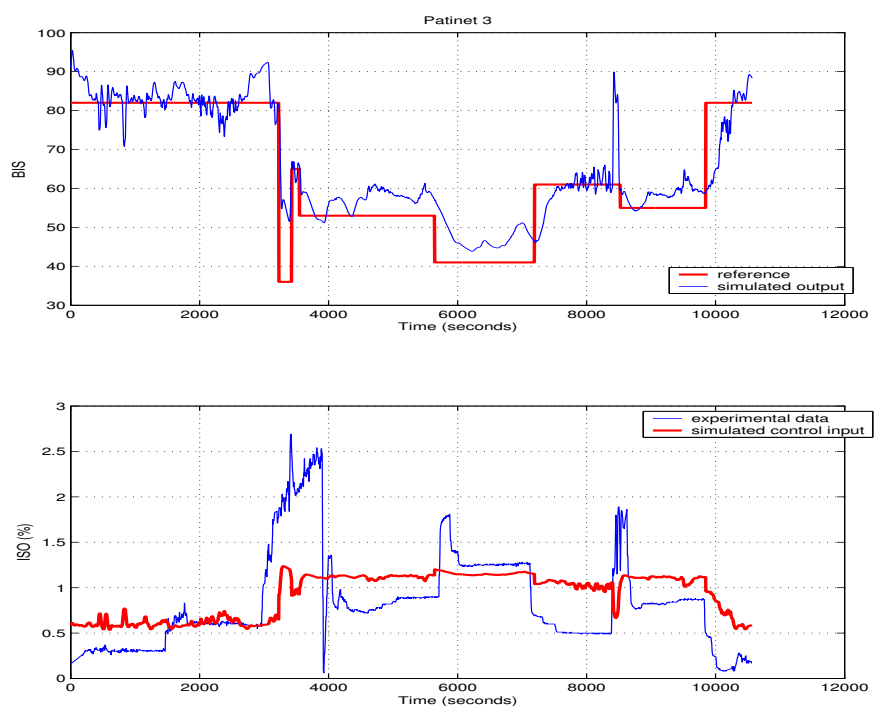

Figure 12: Control simulation result of BIS reference tracking for patient 3: In this 2-by-1 plot, the upper plot shows the desired BIS reference trajectory (red line) and the control simulation output (blue line); the lower plot represents the isoflurane control input (red line) (in \%volume concentration) and the isoflurane measurements from the associated clinical trial (blue line).

restrictions placed on the realization matrices $A, B$ and $C$, for example it is assumed $A$ is Hurwitz. An additional underlying assumption is that the disturbance satisfies a Lipschitz condition in the time domain with Lipschitz constant $L$. For this system, the aim is to design a controller such that $y(s)$ tracks a given reference signal $r(s)$ according to a desired system model $M(s)$, called the reference model, i.e., we want the output to behave as $y(s)=M(s) r(s)$. We rewrite the input-output relationship using the reference model, that is, we set

$$
y(s)=M(s)(u(s)+\sigma(s))
$$

where in the case of a SISO system

$$
\sigma(s)=\frac{(G(s)-M(s)) u(s)+G(s) d(s)}{M(s)} .
$$

We now consider a related closed-loop reference system given by

$$
\begin{aligned}
y_{r e f}(s) & =M(s)\left(u_{r e f}(s)+\sigma_{r e f}(s)\right) \\
\sigma_{r e f} & =\frac{(G(s)-M(s)) u_{r e f}(s)+G(s) d(s)}{M(s)} \\
\text { and } u_{r e f}(s) & =K(s)\left(r(s)-\sigma_{r e f}(s)\right)
\end{aligned}
$$

where in this setting $K(s)$ is a filter designed to attenuate high frequencies in the control channel.

To attain BIBO stability of the closed-loop reference system, $M(s)$ and $K(s)$ must be chosen such that

$$
H(s):=\frac{G(s) M(s)}{K(s) G(s)+(1-K(s)) M(s)}
$$

is stable and the bound

$$
L \cdot\|H(s)(1-K(s))\|_{\mathcal{L}_{1}}<1
$$

holds, where $L$ is the Lipschitz constant for the disturbance term. A parameter adaptation law is designed to estimate and adapt to the difference between the response to the reference system, $M(s)$, and the response 

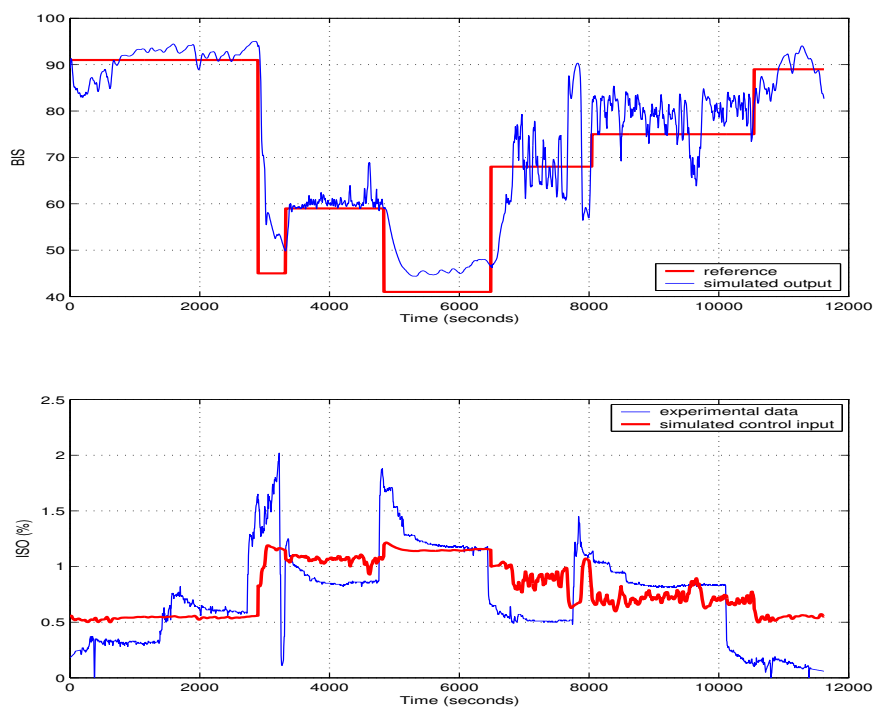

Figure 13: Patient 3 controller with patient 1 model and data for BIS reference tracking: As in the preceding figure, the upper plot represents the desired BIS reference trajectory (red line) and the control simulation output (blue line); the lower plot represents the isoflurane control input (red line) (in \%volume concentration) and the isoflurane measurements from the associated clinical trial (blue line).

to the "true" system given by $G(s)$ (or the difference between $\sigma(s)$ and $\sigma_{r e f}(s)$ ). The adaptation law requires a designed gain term $\Gamma$, and a Lyapunov term $P$ resulting from solving a Lyapunov inequality for the reference system $M(s)$. These terms are used to define the adaptation law $\frac{d \tilde{\sigma}(t)}{d t}$, which leads directly to the $\mathcal{L}_{1}$-adaptive feedback control law

$$
u(s)=K(s)(r(s)-\tilde{\sigma}(s)),
$$

from which the goal is to achieve uniform boundedness of the tracking error, that is,

$$
\|\tilde{y}(t)-y(t)\|_{\mathcal{L}_{\infty}} \leq c(\Gamma P)^{-\frac{1}{2}}
$$

for some computable constant $c$. For further details regarding the $\mathcal{L}_{1}$-adaptive architecture, see [13].

In our preliminary applications of $\mathcal{L}_{1}$-adaptive methods, case study data corresponding to patient BIS values below 70 are used, that is we consider only the sedated portion of the data. Nominal LTI models $(G(s))$ were identified for each patient; these models were third and fourth order. $\mathcal{L}_{1}$-adaptive controllers were designed based on simple first order reference models, $M(s)$, and first order filters, $K(s)$. Parameter adaptation laws were then constructed accordingly and implemented. For example, for a SISO first order reference model such as $M(s)=\frac{m}{s+m}$, the predictor and adaptation laws are of the form

$$
\begin{aligned}
\frac{d \tilde{y}(t)}{d t} & =-m \tilde{y}(t)+m(u(t)+\tilde{\sigma}(t)) \\
\frac{d \tilde{\sigma}(t)}{d t} & =\Gamma \cdot \Pi(\tilde{\sigma}(t),-m P(\tilde{y}(t)-y(t))),
\end{aligned}
$$

where $\Pi$ is a least-squares type projection operator. One example of the resulting BIS and MAP responses is given in Figure 14; this design incorporates both the isoflurane and disturbance inputs. Our initial goal here was conservative: to reach the desired BIS levels in two minutes with little error. Recall that in this case the feedback controller would be implemented following induction to the sedated state performed by an anesthesiologist, so the reference signal $r(t)$ is the latter portion (approximately $0.67 \%$ ) of that used in the LPV designs; however note that the response around the sedation threshold of 70 is fairly smooth.

To assess robustness, controllers designed for one patient model were implemented using the other patients' data. For example, the results of implementing the patient 1 controller on the patient 1,5 and 6 

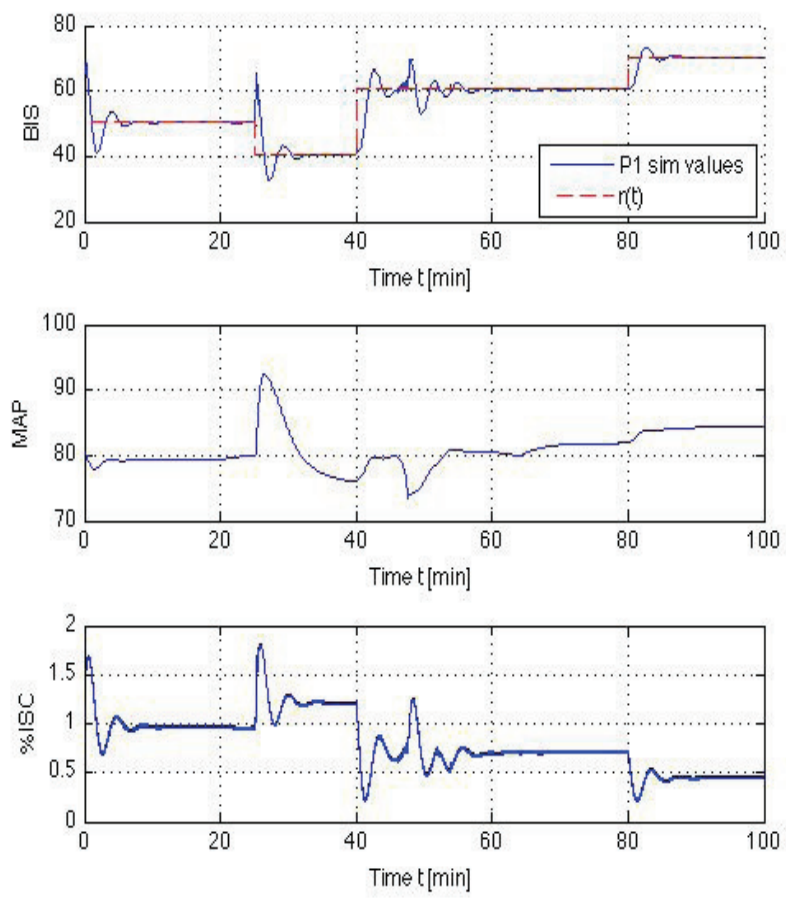

Figure 14: $\mathcal{L}_{1}$-adaptive control: Patient 1 controller with patient 1 model and data. In the upper plot, the desired BIS trajectory and the achieved closed-loop BIS trajectory are given. The center plot gives the achieved closed-loop MAP response; controller specifications are for MAP responses to remain in the 60-100 $\mathrm{mmHg}$ range. The lower plot shows the control effort required to attain the upper plot responses, that is, the resulting Isoflurane needed to track the reference trajectory. 
models and data is shown in Figure 15. The output responses from the patient 5 and 6 models were essentially the same as that of the patient 1 model. However, the patient 6 model required more isoflurane to attain the same reference tracking results. Normalized residual tracking errors were in the $1-5 \%$ range (as compared to the $5-10 \%$ residual tracking errors found over the same range of data using the LPV controllers).
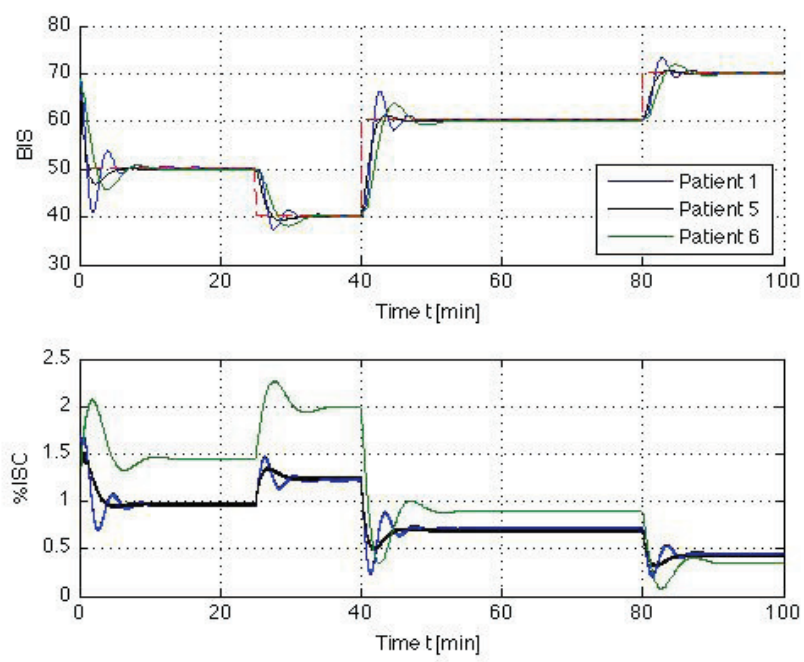

Figure 15: $\mathcal{L}_{1}$-adaptive control: Patient 1 controller with data and models for patients 1,5 and 6 . In the upper plot, the desired BIS trajectory indicated by the dashed line (red), and resulting closed-loop BIS trajectories for patients 1,5 and 6 are indicated by the solid lines (blue, black and green, respectively). The lower plot shows the control effort required to attain the upper plot responses, that is, the resulting Isoflurane needed for each patient model to track the reference trajectory.

Recent designs have included modifications to the $\mathcal{L}_{1}$-adaptive control architecture to address a variety of performance issues, and extended controller operation regimes to incorporate switching and safety mechanisms. The results of these modifications are presented in [41, 42].

\section{Conclusions}

There are clear limitations presented by the standard compartment modeling paradigm when considered for the purpose of closed-loop control design for pharmacodynamics. PK-PD models are inherently singleoutput, and in most cases single input, thus the response of multiple (in particular, coupled) patient output indicators to multi-drug treatments and disturbances can not be captured. Most PK models represent nonnegative system responses; given that for many pharmacological agents these are at best grey-box models this model structure imposes an additional challenge on the control design process which may not be necessary. Further, there are no direct mechanisms for capturing inter- or intra-patient variability, or model uncertainty with the PK-PD model structure.

As a means of completing multivariable control synthesis for a case study problem in anesthetic pharmacodynamics, we have used $\mathcal{H}_{\infty}$ gain-scheduling or LPV techniques, and $\mathcal{L}_{1}$-adaptive methods. These approaches allowed us to include disturbance inputs in our models, and demonstrated some robustness to inter- and intra-patient variations. Multi-drug regimens can be directly incorporated into these underlying model structures when used for control synthesis. Future efforts for additional modeling and control of anesthesia response, suggested by our results and those of our peers thus include continued construction of multi-drug MIMO models, which could be completed using identification-based techniques, as discussed in this paper; design of multi-objective control strategies such as that considered in [76], for example, feedback control strategies aimed at optimizing dosing and administration of anesthetic agents affecting sedation while 
simultaneously regulating hemodynamic functions, and further incorporating robustness analysis and design objectives. Additional studies to determine whether central models exist for patient groups, such as specific population groups, is suggested based on our preliminary cross-validation results and some of the anesthetic pharmacodynamics literature [47, 71, 65, 19, 54].

To move forward with any general implementations of closed-loop anesthesia control, the underlying model structure on which the control synthesis is based must be multivariable, and lend itself to incorporating adaptive and robust control methods. Initial implementation will require the design of enable/disable mechanisms and protocols for the attending anesthesiologist. There will be a doctor-in-the-loop; implementations must be made accordingly for this setting. Similarly, the use of closed-loop insulin delivery devices will also need to account for the patient-in-the-loop. These latter considerations may require a human factors or situational awareness perspective (see, e.g., [44, 79]).

\section{Acknowledgements}

The anesthesia work presented here was the result of many collaborations with students and colleagues. The author would like to thank Dr. Marc Bloom, Evgeny Kharisov, Hui-Hung Lin, Matthew Ralph and Naira Hovakimyan for their many contributions to and conversations regarding the anesthesia control studies discussed herein. This work has been partially supported by the National Science Foundation, grant numbers ECS-0725708 and CNS-1330077, and the Chittenden Foundation.

\section{References}

[1] J.P. Abenstein and M.A. Warner. Anesthesia providers, patient outcomes, and costs. Anesth. Analg., 82:1273-1283, 1996.

[2] A.R. Absalom, N. Sutcliffe, and G.N. Kenny. Closed-loop control of anesthesia using bispectral index: performance assessment in patients undergoing major orthopedic surgery under combined general and regional anesthesia. Anesthesiology, 96:67-73, 2002.

[3] P. Apkarian and P. Gahinet. A convex characterization of gain-scheduled H-infinity controllers. IEEE Trans. on Automatic Control, 1995. Vol. 40, No. 5.

[4] C. L Beck, J.C. Doyle, and K. Glover. Model reduction of multi-dimensional and uncertain systems. IEEE Trans. on Automatic Control, 41(10), 1996.

[5] C. L. Beck, H.H. Lin, and M. Bloom. Modeling and control of anesthetic pharmacodynamics. In G. Garcia S. Tarbouriech, I. Queinnec and S. Niculescu, editors, Biology and Control Theory, Current Challenges. Springer-Verlag Lecture Note Series, 2007.

[6] G. Becker and A. Packard. Robust performance of linear parametrically varying systems using parametrically-depend linear feedback. Systems and Control Letters, 23(3):205-215, Sept. 1994.

[7] G. S. Becker. Quadratic Stability and Performance of Linear Parameter Dependent Systems. PhD thesis, University of California at Berkeley, Nov. 1993.

[8] B. W. Bequette. Challenges and recent progress in the development of a closed-loop artificial pancreas. Annu. Rev. Control, 32:255-266, 2012.

[9] B.W. Bequette. Glucose clamp algorithms and insulin time-action profiles. Journal of Diabetes Science and Technology, 3(5):1005-1013, 2009.

[10] B.W. Bequette. Algorithms for a closed-loop artificial pancreas: The case for model predictive control. Journal of Diabetes Science and Technology, 7(6):1632-1643, 2013.

[11] R. Berne, M. N. Levy, B. M. Koeppen, and B. A. Stanton. Physiology. Mosby, Inc., St. Louis, MI, 4th edition, 1998. 
[12] R.G. Bickford. Automatic electroencephalographic control of anesthesia (servo-anesthesia). Electroenceph. Clin. Neurophys., 3:83-86, 1951.

[13] C. Cao and N. Hovakimyan. Design and analysis of a novel $\mathcal{L}_{1}$ adaptive control architecture with guaranteed transient performance. IEEE Trans. on Automatic Control, 53(2):586-591, 2008.

[14] P. Colmegna and R.S. Sanchez Pena. Linear parameter-varying control to minimize risks in type 1 diabetes. In Proceedings of the IFAC 19th World Congress, 2014.

[15] A. Dahan, D. Nieuwenhuijs, E. Olofsen, E. Sarton, R. Romberg, and L. Teppema. Response surface modeling of alfentanil-sevoflurane interaction on cardiorespiratory control and bispectral index. Anesthesiology, 94(6):982-991, 2001.

[16] O. Dale and B. R. Brown Jr. Clinical pharmacokinetics of the inhalational anaesthetics. Clinical Pharmacokinetics, 12:145-167, 1987.

[17] M. Derighetti, C. W. Frei, M. Buob, A. M. Zbinden, and T. W. Schnider. Modeling the effect of surgical stimulation on mean arterial blood pressure. In Proceedings of the 1997 19th Annual International Conference of the IEEE Engineering in Medicine and Biology Society, volume 5, pages 2172-2175, 1997.

[18] G. E. Dullerud and Fernando Paganini. A Course in Robust Control Theory: A Convex Approach. Springer-Verlag, New York, 2000.

[19] T.D. Egan, B. Huizinga, S.K. Gupta, R.L. Jaarsma, R.J. Sperry, J.B. Yee, and K.T. Muir. Remifentanil pharmacokinetics in obese versus lean patients. Anesthesiology, 89(3):562-573, 1998.

[20] E. I. Eger II. Isoflurance: A review. Anesthesiology, 55(5):559-576, Nov. 1981.

[21] E. I. Eger II. Current and future perspectives on inhaled anesthetics. Pharmacotherapy, 18(5):895-910, 1998.

[22] F. H. El-Khatib, J. Jiang, R.G. Gerrity, and E.R. Damiano. Pharmacodynamics and stability of subcutaneously infused glucagon in a type 1 diabetic swine model in vivo. Diabetes Technology 83 Therapeutics, $9(2): 135-124,2007$.

[23] R.T. Faghih, K. Savla, M. A. Dahleh, and E.N. Brown. A feedback control model for cortisol secretion. In Proceedings of the Annual International Conference of the IEEE Engineering in Medicine and Biology Society, 2011.

[24] C. W. Frei, M. Derighetti, M. Morari, A. H. Glattfelder, and A. M. Zbinden. Improving regulation of mean arterial blood pressure during anesthesia through estimates of surgery effects. IEEE Trans. on Biomed. Eng., 47(11):1456-1464, Nov. 2000.

[25] C. W. Frei, A. Gentilini, M. Derighetti, A. H. Glattfelder, M. Morari, T. W. Schnider, and A. M. Zbinden. Automation in anesthesia. In Proc. American Control Conference, pages 1258-1263, 1999.

[26] A. Gentilini, C. W. Frei, A. H. Glattfelder, M. Morari, T. J. Sieber, R. Wymann, T. W. Schnider, and A. M. Zbinden. Multi-tasked closed loop control in anesthesia. IEEE Eng. in Med. and Bio. Magazine, 20(1):39-53, Jan. 2001.

[27] A. Gentilini, M. Rossoni-Gerosa, C. Frei, R. Wymann, M. Morari, A. Zbinden, and T. Schnider. Modeling and closed loop control of hypnosis by means of bispectral index (BIS) with isoflurane. IEEE Trans. on Biomed. Eng., 48(8):874-889, Aug. 2001.

[28] A. Gentilini, C. Schaniel, M. Morari, C. Bieniok, R. Wymann, and T. Schnider. A new paradigm for the closed-loop intraoperative administration of analgesics in humans. IEEE Trans. on Biomed. Eng., 49(4):289-299, April 2002. 
[29] P. S. Glass, M. Bloom, L. Kearse, C. Rosow, P. Sebel, and P. Manberg. Bispectral analysis measures sedation and memory effects of propofol, midazolam, isoflurane, and alfentanil in healthy volunteers. Anesthesiology, 86(4):836-847, April 1997.

[30] S. Goutelle, M. Maurin, F. Tougier, X. Barbaut, L. Bourguignon, M. Cusher, and P. Maire. The Hill equation: a review of its capabilities in pharmacological modeling. Fundamental and Clinical Pharmacology, 22:633-648, 2008.

[31] W. Haddad, J. Bailey, T. Hayakawa, and N. Hovakimyan. Neural network adaptive output feedback control for intensive care unit sedation and operating room hypnosis. IEEE Transactions on Neural Networks, 18(4):1049-1066, 2007.

[32] A. V. Hill. The possible effects of the aggregations of the molecules of haemoglobin on its dissociation curves. J. Physiology, 40:iv-vii, 1910.

[33] E. I. Eger II, L. J. Saidman, and Editors R. N. Westhorpe. The Wondrous Story of Anesthesia. Springer Science and Business Media, 2014.

[34] F. J Doyle III, L. M. Huyett, J. B. Lee, H. C. Zisser, and E. Dassau. Closed-loop artificial pancreas systems: Engineering the algorithms. Diabetes Care, 37:1191-1197, 2014.

[35] J. O. Johnson and S. E. Kern. Bispectral electroencephalographic analysis for patient monitoring during anesthesia. Advances in Anesthesia, pages 61-81, 1999.

[36] V. Jonsson, N. Matni, and R. Murray. Synthesizing combination therapies for evolutionary dynamics of disease for nonlinear pharmacodynamics. In Proceedings of the IEEE Conference on Decision and Control, 2014.

[37] V. Jonsson, A. Rantzer, and R. Murray. A scalable formulation for engineering combination therapies for evolutionary dynamics of disease. In Proceedings of the American Control Conference, 2014.

[38] B. Kabon and A. Kurz. Optimal perioperative oxygen administration. Current Opinion in Anesth., 19:11-18, 2006.

[39] A. H. Kadish. Automation control of blood sugar: A servomechanism for glucose monitoring and control. Transactions American Society for Artificial Internal Organs, 9:363-367, 1963.

[40] S.E. Kern, G. Xie, J.L. White, and T.D. Egan. A response surface analysis of propofol-remifentanil pharmacodynamic interaction in volunteers. Anesthesiology, 100(6):1373-1381, 2004.

[41] E. Kharisov, C. L. Beck, and M. Bloom. Control of patient response to anesthesia using $\mathcal{L}_{1}$-adaptive methods. In $8^{\text {th }}$ IFAC Symposium on Biological and Medical Systems, 2012.

[42] E. Kharisov, C. L. Beck, and M. Bloom. Regulation of anesthesia delivery using $\mathcal{L}_{1}$-adaptive methods. In SIAM Conference on Control and its Applications, 2013.

[43] E. Kharisov, C. L. Beck, and M. Bloom. Design of $\mathcal{L}_{1}$-adaptive controllers for human patient anesthesia. Control Engineering Practice, 2014. Submitted.

[44] A. Kirlik and R. Strauss. Situation awareness as judgment I: Theoretical framework, modeling, and quantitative measurement. International Journal of Industrial Ergonomics: Special Issue on New Insights in Human Performance and Decision Making, pages 463-474, 2006.

[45] A. Kurz and D. Sessler. Opioid-induced bowel dysfunction: pathophysiology and potential new therapies. Drugs, 63(7):649-671, 2003.

[46] J. Lang. Awakening. The Atlantic, 2013. 
[47] E. B. Liem, C-M. Lin, M-I. Suleman, A.G. Doufas, and D. I. Sessler. Increased anesthetic requirement in subjects with naturally red hair. Anesthesiology, 2002. 97:A-77.

[48] H. H. Lin. Multivariable Modeling and Control of the Response to Anesthesia. PhD thesis, University of Illinois at Urbana-Champaign, May 2006.

[49] H. H. Lin, C. L. Beck, and M. Bloom. Multivariable LPV control of anesthesia delivery during surgery. In $A C C$, pages 825-831, 2008.

[50] H. H. Lin, C. L. Beck, and M. J. Bloom. On the use of multivariable piecewise-linear models for predicting human response to anesthesia. IEEE Trans. on Biomedical Engineering, 51(11):1876-1887, 2004.

[51] L. Ljung. System Identification: Theory for the User. Prentice Hall PTR, Upper Saddle River, NJ, 2nd edition, 1999.

[52] S.C. Manyam, D.K. Gupta, K.B. Johnson, J.L. Shite, N.L. Pace, D.R. Westenskow, and T.D. Egan. Opioid-volatile anesthetic synergy: a response surface model with remifentanil and sevoflurane as prototypes. Anesthesiology, 105(2):267-278, 2006.

[53] R. Martin and K. L. Teo. Optimal Control of Drug Administration in Cancer Chemotherapy. World Scientific, 1993.

[54] C.F. Minto, T.W. Schnider, T.D. Egan, E.J. Youngs, H.J. Lemmens, P.L. Gambus, V. Billard, J.F. Hoke, K.H. Moore, D.J. Hermann, K.T. Muir, J.W. Mandema, and S.L. Shafer. Influence of age and gender of the pharmacokinetics and pharmacodynamics of remifentanil. I. model development. Anesthesiology, 86(1):10-23, 1997.

[55] C.F. Minto, T.W. Schnider, T.G. Short, K.M. Gregg, A. Gentilini, and S.L. Shafer. Response surface model for anesthetic drug interactions. Anesthesiology, 92(6):1603-1616, 2000.

[56] A. Morley, J. Derrick, P. Mainland, B.B. Lee, and T.G. Short. Closed loop control of anaesthesia: an assessment of the bispectral index as the target of control. Anaesthesia, 55(10):953-959, 2000.

[57] E. Mortier, M. Struys, T. De Smet, L. Versichelen, and G. Rolly. Closed-loop controlled administration of propofol using bispectral analysis. Anesthesia, 53(8):749-754, 1998.

[58] E. Olofsen and A. Dahan. The dynamic relationship between end-tidal sevoflurane and isoflurane concentrations and bispectral index and spectral edge frequency of the electroencephalogram. Anesthesiology, 90(5):1345-1353, May 1999.

[59] P.A. Orlando, R.A. Gatenby, and J.S. Brown. Cancer treatment as a game: integrating evolutionary game theory into the optimal control of chemotherapy. Physical Biology, 9, 2012.

[60] A. Packard. Gain scheduling via linear fraction transformations. System and Control Letters, 22(2):7992, Feb. 1994.

[61] R.S. Parker, F. J. Doyle, and N. A. Peppas. A model-based algorithm for blood glucose control in type 1 diabetic patients. IEEE Trans. on Biomed. Engineering, 46(2), 1999.

[62] M. Ralph, C. L. Beck, and M. Bloom. $\mathcal{L}_{1}$-adaptive methods for control of patient response to anesthesia. In $A C C$, pages 1729-1735, 2011.

[63] S. J. Russell, F. H. El-Khatib, D. M. Nathan, and E. R. Damiano. Efficacy determinants of subcutaneous microdose glucagon during closed-loop control. Journal of Diabetes Science and Technology, 4, 2010.

[64] T. W. Schnider, C. F. Minto, and D. R. Stanski. The effect compartment concept in pharmacodynamic modelling. Anesthetic Pharmacology Review, 2(3):204-213, 1994. 
[65] T.W. Schnider, C.F. Minto, S. L. Shafer, P.L. Gambus, C. Andresen, D.B. Goodale, and E.J. Youngs. The influence of age on propofol pharmacodynamics. Anesthesiology, 90(6):1502-1516, 1999.

[66] H. Schwilden and H. Stoeckel. Effective therapeutic infusions produced by closed-loop feedback control of methohexital administration during total intravenous anesthesia with fentanyl. Anesthesiology, 73(2):225-229, Aug. 1990.

[67] H. Schwilden and H. Stoeckel. Closed-loop feedback controlled administration of alfentanil during alfentanil-nitrous oxide anaesthesia. British Journal of Anaesthesia, pages 389-393, 1993.

[68] H. Schwilden, H. Stoeckel, and J. Schüttler. Closed-loop feedback control of propofol anaesthesia by quantitative EEG analysis in humans. British Journal of Anaesthesia, pages 290-296, 1989.

[69] P. Sebel, E. Lang, I. J. Rampil, P. F. White, R. Cork an d M. Jopling, N. T. Smith, P. S. Glass, and P. Manberg. A multicenter study for bispectral electroencephalogram analysis for monitoring anesthetic effect. Anesthesia and Analgesia, pages 891-899, 1997.

[70] P. S. Sebel. Bispectral monitoring technology: Clinical applications. In T.H. Stanley and T.D. Egan, editors, Anesthesia for the New Millennium - Modern Anesthetic Clinical Pharmacology, pages 99-104. Kluwer Academic Publishers, 1999.

[71] S. L. Shafer. The pharmacology of anesthetic drugs in elderly patients. Anesthesiol. Clin. North America, 18(1):1-29, 2000.

[72] S. Shahruz and S. Behtash. Design of controllers for linear parameter varying systems by the gain scheduling technique. Journal of Mathematical Analysis and Applications, 168(1):195-217, 1992.

[73] J. Shamma. An overview of LPV systems. In J. Mohammadpour and C. Scherer, editors, Control of Linear Parameter Varying Systems with Applications. Springer, 2012.

[74] J. Shamma and M. Athans. Guaranteed properties of gain scheduled control of linear parameter-varying plants. Automatica, 27(3):559-564, 1991.

[75] J. C. Sigl and N. G. Chamoun. An introduction to bispectral analysis for the electroencephalogram. Journal of Clinical Monitoring, 10(6):392-404, Nov. 1994.

[76] K. Soltesz, G. A. Dumont, K.van Heusden, T. Hgglund, and J. M.Ansermino. Simulated mid-ranging control of propofol and remifentanil using eeg-measured hypnotic depth of anesthesia. In Proceedings of the IEEE Conference on Decision and Control, 2012.

[77] D. R. Stanksi. Monitoring Depth of Anesthesia. Churchill Livingston, New York, 3rd edition, 1990. in Anesthesia, ED R. D. Miller.

[78] G. M. Steil and M. F. Saad. Automated insulin delivery for type 1 diabetes. Current Opinion in Endocrinology \& Diabetes, 13:205-211, 2006.

[79] R. Strauss and A. Kirlik. Situation awareness as judgment II: Experimental evaluation and demonstration. International Journal of Industrial Ergonomics: Special Issue on New Insights in Human Performance and Decision Making, 36:475-484, 2006.

[80] G.W. Swan and T. L. Vincent. Optimal control analysis in the chemotherapy of IgG multiple myeloma. Bull. Math. Biol., 39:317-337, 1977.

[81] K. van Heusden, E. Dassau, H.C. Zisser, D.E. Seborg, and F. J. Doyle. Control-relevant models for glucose control using a priori patient characteristics. IEEE Trans. on Biomedical Engineering, 59(7):1839$1849,2012$. 
[82] K. van Heusden, G. A. Dumont, K. Soltesz, C. L. Petersen, A. Umedaly, N. West, and J. M. Ansermino. Design and clinical evaluation of robust pid control of propofol anesthesia in children. IEEE Transactions on Control Systems Technology, 22:491?501, 2014.

[83] M. Viberg, B. Wahlberg, and B. Ottersten. Analysis of state space system identification methods based on instrumental variables and subspace fitting. Automatica, 33(9):1603-1616, 1997.

[84] M. von Spreckelsen and B. Bromm. Estimation of single-evoked cerebral potentials by means of parametric modeling and kalman filtering. IEEE Trans. on Biomed. Eng., 35(9):691-700, Sept. 1988.

[85] J. G. Wagner. Kinetics of pharmacologic response. i. proposed relationships between response and drug concentration in the intact animal and man. Journal of Theoretical Biology, 20:173-201, 1968.

[86] N. Yasuda, S. H. Lockhart, E. I. Eger II, R. B. Weiskopf, J. Liu, M. Laster, S. Taheri, and N. A. Peterson. Comparison of kinetics of sevoflurane and isoflurane in humans. Anesthesia Analgesia, pages 316-324, 1991.

[87] G. Zames. On the input-output stability of nonlinear time-varying feedback systems, parts i and ii. IEEE Trans. on Automatic Control, pages 228-238 and 465-476, 1966.

[88] R. Zurakowski. Nonlinear observer output-feedback MPC treatment scheduling for HIV. Biomedical Engineering Online, 10(1), 2011. 